\title{
SUBGROUPS OF GROUPS OF CENTRAL TYPE
}

\author{
BY \\ KATHLEEN M. TIMMER
}

ABSTRACT. Let $\lambda$ be a linear character on the center $Z$ of a finite group $Z$ of a finite group $H$, such that

(1) $\lambda^{H}=\sum_{i=1}^{p} \phi_{i}(1) \phi_{i}$ where the $\phi_{i}$ 's are inequivalent irreducible characters on $H$ of the same degree, and

(2) if $\sum_{i-1}^{i} m_{i} \phi_{i}(x)=0$ for some $x \in H$ and nonnegative integers $m_{b}$ then either $\phi_{i}(x)=0$ for all $i$ or $m_{i}=m_{j}$ for all $i, j$.

The object of the paper is to describe finite groups which satisfy conditions (1) and (2) in terms of the multiplication of the group. If $S$ is a $p$ Sylow subgroup of the group $H$, and $R=S \cdot Z$, then $H$ satisfies conditions (1) and (2) if and only if

(a) $\left\{x \in H: x^{-1} h^{-1} x h \in Z \Rightarrow \lambda\left(x^{-1} h^{-1} x h\right)=1, h \in H\right\} / Z$ consists of elements of order a power of $p$ in $H / Z$, and these elements form $p$ conjugacy classes of $H / Z$, and

(b) the elements of $\left\{x \in R: x^{-1} r^{-1} x r \in Z \Rightarrow \lambda\left(x^{-1} r^{-1} x r\right)=1, r \in R\right\} / Z$ form $p$ conjugacy classes of $R / Z$.

Introduction. Let $G$ be a finite group with center $Z$. In [3] F. R. DeMeyer and G. J. Janusz called $G$ a group of central type if there is an irreducible (complex) character $\chi$ on $G$ such that $\chi(1)^{2}=[G: Z]$. Groups of central type arise in Schur's theory of projective representations [5, pp. 628-655] and the general Galois theory of rings [1].

We study groups which appear as normal subgroups of index $p$ for some prime $p$ in groups of central type. Let $H$ be a finite group with center $Z$, and let $p$ be a prime. Let $\lambda$ be a linear character on the center $Z$ of a finite group $H$, such that $\lambda^{H}=\sum_{i=1}^{p} \phi_{i}(1) \phi_{i}$ where the $\phi_{i}$ 's are inequivalent irreducible characters on $H$ of the same degree. Assume that if $\sum_{i=1}^{p} m_{i} \phi_{i}(x)=0$ for nonnegative integers $m_{b}$, then either $\phi_{i}(x)=0$ for all $i$ or $m_{i}=m_{j}$ for all $i, j$. We call a group satisfying these conditions $p$-special with respect to $\lambda$.

We show that if $H$ is a normal subgroup of index $p$ in a group $G$ of central type, then either $H$ is of central type or $H$ is p-special (Theorem 2.1). We next give necessary and sufficient conditions on a $p$-special group $H$ that it be a normal subgroup of index $p$ in a group of central type (Theorem 2.2).

We then examine some properties of $p$-special groups. For a group $H$, let $\mathrm{Cl}_{H}(x)$ be the conjugacy class in $H$ containing $x$. Let $Z$ be the center of $H$ and let $\lambda$ be a linear character on $Z$. Define

$$
T(H, \lambda)=\left\{x \in H: x^{-1} \mathrm{Cl}_{H}(x) \cap Z \subseteq \operatorname{kernel}(\lambda)\right\} .
$$

Received by the editors June 6, 1972.

AMS (MOS) subject classifications (1969). Primary 20C15, 20C25, 20F15, 20C20, 20 C25.

Key words and phrases. Character, representation, group of central type, character of large degree, projective representation, projective group algebra. 
If $S$ is a $p$ Sylow subgroup of $H$, let $R=S \cdot Z$ and define

$$
T(R, \lambda)=\left\{x \in R: x^{-1} \mathrm{Cl}_{R}(x) \cap Z \subseteq \operatorname{kernel}(\lambda)\right\} .
$$

We show (Corollary 2.26) that if $H$ is a finite group with center $Z$, then $H$ is $p$ special with respect to $\lambda$ if and only if

(1) every element of $T(H, \lambda) / Z$ has order a power of $p$ and $T(H, \lambda) / Z$ consists of $p$ conjugacy classes of $H / Z$, and

(2) $T(R, \lambda) / Z$ consists of $p$ conjugacy classes of $R / Z$.

Additional information is given concerning the set of elements $T(H, \lambda)$ and how it relates to the character on $H$.

Throughout this paper, all groups are finite and all characters are complex. If $H$ is a group, $Z(H)$ denotes the center of $H$. If $x \in H,\langle x\rangle$ denotes the subgroup of $H$, generated by $x$. The conjugacy class of $x$ is denoted by $\mathrm{Cl}_{H}(x)$ or simply by $\mathrm{Cl}(x)$ if there can be no confusion. If $A$ is a subset of $H,[A: 1]$ denotes the number of elements in $A$ and if $A$ and $B$ are two subsets, $[A: B]=[A: 1] /[B: 1]$. A $p$ element is an element whose order is a power of $p$ and a $p$ group is a group in which every element is a $p$ element. If $n$ is any integer and $q$ is any prime $n_{q}$ denotes the $q$ part (or $q$ factor) of $n$. All unexplained terminology and notation is as in Huppert [5].

This paper is the author's doctoral thesis at Colorado State University written under the direction of Professor Frank R. DeMeyer. The author extends her heartfelt thanks to Dr. DeMeyer for his advice and encouragement. She also wishes to express her appreciation to Dr. Gerald J. Janusz of the University of Illinois for his careful reading of the text and for offering many corrections and suggestions.

1. F. R. DeMeyer and G. J. Janusz [3] defined a finite group $G$ with center $Z$ to be of central type if there is an irreducible character $\chi$ on $G$ so that $\chi(1)^{2}=[G: Z]$. They proved the following: If $G$ is a group of central type then there is a 2 -cocycle $\alpha$ on $\bar{G}=G / Z$ so that $K(\bar{G})_{\alpha}$ has center $K$, where $K$ denotes the set of complex numbers. Herbert Pahling [6] showed that if $G$ is a group of central type with center $Z$, then for every $x \in G, x \notin Z$, there is an element $g \in G$ so that $1 \neq x^{-1} g^{-1} x g \in Z$; and conversely, if $[G, G] \cap Z$ is cyclic and for every $x \in G, x \notin Z$, there is an element $g \in G$ so that $1 \neq x^{-1} g^{-1} x g \in Z$, then $G$ is a group of central type. In this section, results will be proved which connect the above results.

Let $G$ be a group with center $Z$ and let $\mathrm{Cl}(x)$ be the conjugacy class in $G$ containing $x$. The condition in Pahling's results suggests the study of the elements $x \in G$, for which $x^{-1} \mathrm{Cl}(x) \cap Z=\{1\}$. In order to make the results of this section as general as later applications require, we study a larger set.

Definition. Let $A$ be a subgroup of the center $Z$ of a group $G$ and let $\lambda$ be a linear character on $A$. Define

$$
T(G, \lambda)=\left\{x \in G: x^{-1} \mathrm{Cl}(x) \cap A \subseteq \operatorname{kernel}(\lambda)\right\} .
$$


If $x \in T(G, \lambda)$, then $\mathrm{Cl}(x) \subseteq T(G, \lambda)$ and $x \cdot a \in T(G, \lambda)$ for all $a \in A$. The results of this section give relationships between the irreducible constituents of $\lambda^{G}$ and the elements of the set $T(G, \lambda)$.

Lemma 1.1. Let $G$ be a group with center $Z$ and let $A$ be a subgroup of $Z$. If $\lambda$ is a linear character on $A$, then there is a 2-cocycle $\alpha$ on $G / Z$ so that the center of the projective group algebra $K(G / A)_{\alpha}$ has dimension $t$ over $K$ where $t$ is the number of conjugacy classes of $G / A$ contained in $T(G) / A$. Moreover, a basis of the center of $K(G / A)_{\alpha}$ consists of elements of the form $\sum_{x \in C} U_{x}$, where $C$ is the natural image in $G / A$ of a conjugacy class of $G$ contained in $T(G, \lambda)$.

We prove Lemma 1.1 first in the case that $\lambda$ restricted to $[G, G] \cap A$ is faithful. We carry out the proof of Lemma 1.1 by a sequence of assertions.

(1.2) If $\gamma \in \bar{G}=G / A$, let $\gamma^{*}$ be an element of $G$, chosen to represent the coset $\gamma$. It is possible to choose the coset representatives in such a way that if $\beta, \gamma \in \bar{G}$ with $\beta$ the natural image of an element of $T(G, \lambda)$, then $\left(\gamma^{-1} \beta \gamma\right)^{*}=\left(\gamma^{*}\right)^{-1} \beta^{*} \gamma^{*}$.

Proof. Let $C_{1}, \ldots, C_{t}$ be distinct conjugacy classes in $\bar{G}$, contained in $T(G, \lambda) / A$, where $C_{1}=\{\overline{1}\}$. Choose $(\overline{1})^{*}=1$. For each $2 \leq i \leq t$, fix an element $\beta \in C_{i}$ and choose $\beta^{*}$. For every $\gamma \in \bar{G}$, define $\left(\gamma^{-1} \beta \gamma\right)^{*}=(g)^{-1} \beta^{*} g$, where $\bar{g}$ $=\gamma$. We must show that $\left(\gamma^{-1} \beta \gamma\right)^{*}$ is well defined.

First of all it is clear that the definition of $\left(\gamma^{-1} \beta \gamma\right)^{*}$ is independent of the choice of $g$, since $A \subseteq Z$. Suppose $\delta^{-1} \beta \delta=\gamma^{-1} \beta \gamma$ for some $\delta \in \bar{G}$. Let $d \in G$, so that $\bar{d}=\delta$. Then $d^{-1} \beta^{*} d=g^{-1} \beta^{*} g \cdot a$ for some $a \in A$, and

$$
a^{-1}=\left(\beta^{*}\right)^{-1}\left(g d^{-1}\right)^{-1} \beta^{*} g d^{-1} \in A \cap[G, G] .
$$

Since $\beta \in C_{i}, \beta^{*} \in T(G, \lambda)$ and $\left(\beta^{*}\right)^{-1}\left(g h^{-1}\right)^{-1} \beta^{*} g h^{-1}=a \in \operatorname{kernel}(\lambda)$. Since $\lambda$ restricted to $[G, G] \cap A$ is assumed to be faithful, $a=1$, and $d^{-1} \beta^{*} d$ $=g^{-1} \beta^{*} g$. Hence $\left(\gamma^{-1} \beta \gamma\right)^{*}$ is well defined. Choose the representatives of other elements of $\bar{G}$ arbitrarily. This completes the proof of (1.2).

Choose coset representatives of the elements of $\bar{G}$ as described in (1.2). We define a 2 -cocycle $\alpha$ on $\bar{G}$ by

$$
\alpha(\delta, \gamma)=\lambda\left(\left((\delta \gamma)^{*}\right)^{-1} \delta^{*} \gamma^{*}\right)
$$

We isolate the following computation.

(1.3) If $\beta$ is in the image of an element in $T(G, \lambda)$, then for every $\gamma \in \bar{G}$, $\alpha\left(\gamma^{-1}, \beta\right) \alpha\left(\gamma^{-1} \beta, \gamma\right)=\lambda\left(\left(\gamma^{-1}\right)^{*} \gamma^{*}\right)$.

Proof. By (1.2), $\left(\gamma^{-1} \beta \gamma\right)^{*}=\left(\gamma^{*}\right)^{-1} \beta^{*} \gamma^{*}$. Then

$$
\begin{aligned}
\alpha\left(\gamma^{-1}, \beta\right) \alpha\left(\gamma^{-1} \beta, \gamma\right) & =\lambda\left(\left(\left(\gamma^{-1} \beta\right)^{*}\right)^{-1}\left(\gamma^{-1}\right)^{*} \beta^{*}\right) \cdot \lambda\left(\left(\left(\gamma^{-1} \beta \gamma\right)^{*}\right)^{-1}\left(\gamma^{-1} \beta\right)^{*} \gamma^{*}\right) \\
& =\lambda\left(\left(\left(\gamma^{-1} \beta\right)^{*}\right)^{-1}\left(\gamma^{-1}\right)^{*} \beta^{*}\right) \cdot \lambda\left(\left(\gamma^{*}\right)^{-1}\left(\beta^{*}\right)^{-1} \gamma^{*}\left(\gamma^{-1} \beta\right)^{*} \gamma^{*}\right) \\
& =\lambda\left(\left(\left(\gamma^{-1} \beta\right)^{*}\right)^{-1}\left(\gamma^{-1}\right)^{*} \beta^{*}\left(\beta^{*}\right)^{-1} \gamma^{*}\left(\gamma^{-1} \beta\right)^{*}\right) \\
& =\lambda\left(\left(\gamma^{-1}\right)^{*} \gamma^{*}\right) .
\end{aligned}
$$


(1.4) Suppose that $\sum_{\gamma \in \bar{G}} c_{\gamma} U_{\gamma}$ is in the center of $K(\bar{G})_{\alpha}$ where $c_{\gamma} \in K$. If $\gamma$ is not an element of the image of $T(G, \lambda)$ in $\bar{G}$, then $c_{y}=0$. If $\gamma$ is in the image of $T(G, \lambda)$, then $c_{\delta^{-1} \gamma \delta}=c_{\gamma}$ for every $\delta \in \bar{G}$.

Proof. $\sum_{\gamma \in \Gamma} c_{\gamma} U_{\gamma}$ is in the center of $K(\bar{G})_{\alpha}$ if and only if for every $\delta \in \bar{G}$,

$$
\begin{aligned}
U_{\delta-1}\left(\sum_{\gamma \in \bar{G}} c_{\gamma} U_{\gamma}\right) U_{\delta} & =U_{\delta-1} U_{\delta}\left(\sum_{\gamma \in \bar{G}} c_{\gamma} U_{\gamma}\right) \\
& =\lambda\left(\delta^{*}\left(\delta^{-1}\right)^{*}\right)\left(\sum_{\gamma \in \bar{G}} c_{\gamma} U_{\gamma}\right) .
\end{aligned}
$$

Computing, we have

$$
\begin{aligned}
U_{\delta-1}\left(\sum_{\gamma \in \bar{G}} c_{\gamma} U_{\gamma}\right) U_{\delta} & =\sum_{\gamma \in \bar{G}} c_{\gamma} \alpha\left(\delta^{-1}, \gamma\right) \alpha\left(\delta^{-1} \gamma, \delta\right) U_{\delta-1} \delta \\
& =\sum_{\gamma \in \bar{G}}\left(c_{\delta \gamma \delta-1}\right) \alpha\left(\delta^{-1}, \delta \gamma \delta^{-1}\right) \alpha\left(\gamma \delta^{-1}, \delta\right) U_{\gamma} .
\end{aligned}
$$

Hence, for every $\delta$ and $\gamma$ in $\bar{G}$,

$$
\left(c_{3 \gamma-1}\right) \alpha\left(\delta^{-1}, \delta \gamma \delta^{-1}\right) \alpha\left(\gamma \delta^{-1}, \delta\right)=\lambda\left(\delta^{*}\left(\delta^{-1}\right)^{*}\right) c_{\gamma} .
$$

If $\gamma$ is not in the image of $T(G, \lambda)$, there is an element $g \in G$, so that $g \gamma^{*} g^{-1}=\gamma^{*} a$ for some $a \neq 1$ in $A$. Let $\delta=\bar{g}$. Then

$$
\begin{aligned}
\alpha\left(\delta^{-1}, \delta \gamma \delta^{-1}\right) \alpha\left(\gamma \delta^{-1}, \delta\right) & =\lambda\left(\left(\left(\gamma \delta^{-1}\right)^{*}\right)^{-1}\left(\delta^{-1}\right)^{*}\left(\delta \gamma \delta^{-1}\right)^{*}\left(\gamma^{*}\right)^{-1}\left(\gamma \delta^{-1}\right)^{*} \delta^{*}\right) \\
& =\lambda\left(\left(\left(\gamma \delta^{-1}\right)^{*}\right)^{-1}\left(\delta^{-1}\right)^{*} \gamma^{*}\left(\gamma^{*}\right)^{-1}\left(\gamma \delta^{-1}\right)^{*} \delta^{*}\right) \\
& =\lambda\left(\left(\delta^{-1}\right)^{*} \delta^{*} \cdot a\right) \\
& \neq \lambda\left(\left(\delta^{-1}\right)^{*} \delta^{*}\right) .
\end{aligned}
$$

Since equation (1.5) must hold, $c_{\gamma}=0$ for every $\gamma$ not in the image of $T(G, \lambda)$.

If $\gamma$ is in the image of $T(G, \lambda)$, then $\delta \gamma \delta^{-1}$ is also and by (1.3), for every $\delta \in \bar{G}$,

$$
\alpha\left(\delta^{-1}, \delta \gamma \delta^{-1}\right) \alpha\left(\gamma \delta^{-1}, \delta\right)=\lambda\left(\left(\delta^{-1}\right)^{*} \delta^{*}\right) .
$$

Thus $c_{8 y-1}=c_{\gamma}$ for every $\delta \in \bar{G}$.

If $C_{1}, \ldots, C_{t}$ are distinct conjugacy classes in $\bar{G}$ contained in $T(G, \lambda) / A$, then the elements $\sum_{\gamma \in C} U_{\gamma}$ for $C=C_{i}$ for $1 \leq i \leq t$ form a linearly independent set of elements in the center of $K(\bar{G})_{\alpha}$. By (1.4) these elements form a basis of the center of $K(\bar{G})_{\alpha}$. This completes the proof of Lemma 1.1 when $\lambda$ restricted to $[G, G] \cap A$ is faithful.

If $\lambda$ restricted to $[G, G] \cap A$ is not faithful, let $N=[G, G] \cap \operatorname{kernel}(\lambda)$. Let $G^{\prime}=G / N, A^{\prime}=A / N$ and let $\lambda^{\prime}$ be a linear character on $G^{\prime}$ defined by $\lambda^{\prime}(a N)=\lambda(a)$ for any $a \in a N$. Then $T\left(G^{\prime}, \lambda^{\prime}\right)$ in $G^{\prime}$ is the natural image of $T(G, \lambda)$ and the number of conjugacy classes of $G^{\prime} / A^{\prime}$ contained in $T\left(G^{\prime}, \lambda^{\prime}\right) / A^{\prime}$ is the same as the number of classes of $G / A$ in $T(G, \lambda) / A$. Since $G / A$ is isomorphic to $G^{\prime} / A^{\prime}$, if $\alpha^{\prime}$ is a 2-cocycle on $G^{\prime} / A^{\prime}$ as defined in the previous case. 
then there is a corresponding 2-cocycle $\alpha$ on $G / A$, so that $K(G / A)_{\alpha}$ and $K\left(G^{\prime} / A^{\prime}\right)_{\alpha^{\prime}}$ are isomorphic $K$-algebras. This completes the proof of Lemma 1.1.

Lemma 1.1 allows us to count the number of inequivalent irreducible constituents of $\lambda^{G}$, where $\lambda$ is a linear character on a subgroup $A$ of the center of $G$.

(1.6) The number of inequivalent irreducible constituents of $\lambda^{G}$ is $t$, the number of conjugacy classes of $G / A$ contained in $T(G, \lambda) / A$.

Proof. Let $\alpha$ be a 2-cocycle on $G / A$ as defined in the proof of Lemma 1.1. By [4, pp. 163-179], $K(G / A)_{\alpha}$ is isomorphic to $\sum_{i=1}^{t} \operatorname{Hom}_{K}\left(M_{i}, M_{i}\right)$ where $M_{i}$ is an irreducible left $K(G / A)_{\alpha}$ module.

For each $i$, let $T_{i}^{*}$ be a projective representation of $G / A$ corresponding to $M_{i}$. If $g \in G$ and $\bar{g}$ is its image in $G / A$, then $g=(\bar{g})^{*} a(g)$ for some element $a(g) \in A$. Define $T_{i}(g)=\lambda(a(g)) T_{i}^{*}(\bar{g})$. Let $g$ and $d$ be elements of $G$. Then $g=(\bar{g})^{*} a(g), d=(\bar{d})^{*} a(d), g d=(\bar{g} \bar{d})^{*} a(g d)$ and

$$
\begin{aligned}
T_{i}(g) T_{i}(d) & =\lambda(a(g)) T_{i}^{*}(\bar{g}) \lambda(a(d)) T_{i}^{*}(\bar{d}) \\
& =\lambda(a(g) a(d)) \alpha(\bar{g}, \bar{d}) T_{i}^{*}(\bar{g} \bar{d}) \\
& =\lambda(a(g) a(d)) \lambda\left(\left((\bar{g} \bar{d})^{*}\right)^{-1}(\bar{g})^{*}(\bar{d})^{*}\right) T_{i}^{*}(\bar{g} \bar{d}) \\
& =\lambda(a(g d)) T_{i}^{*}(\bar{g} \bar{d})=T_{i}(g d) .
\end{aligned}
$$

Hence $T_{i}$ is an ordinary representation of $G$. If $\phi_{i}(g)=\operatorname{trace}\left(T_{i}(g)\right)$ for $g \in G$, then $\phi_{i}$ is an irreducible character on $G$ for $1 \leq i \leq t$, and $\left.\phi_{i}\right|_{A}=\phi_{i}(1) \lambda$.

Let $\zeta$ be an irreducible constituent of $\lambda^{G}$ and let $M$ be a corresponding $K G$ module. Since $\left.\zeta\right|_{A}=\zeta(1) \lambda, M$ is an irreducible left $K(G / A)_{\alpha}$ module. By [4, Theorem 25.10, p. 166], $M$ is isomorphic to a minimal left ideal of $K(G / A)_{\alpha}$ and $M$ is isomorphic to $M_{i}$ for some $1 \leq i \leq t$. Thus if $\zeta$ is an irreducible constituent of $\lambda^{G}$, then $\zeta=\phi_{i}$ for some $i$.

Let $\phi_{1}, \ldots, \phi_{u}$ be a maximum number of inequivalent characters from the set $\left\{\phi_{i} \mid 1 \leq i \leq t\right\}$. Since $\left(\left.\phi_{i}\right|_{A}, \lambda\right)=\left(\lambda^{G}, \phi_{i}\right)=\phi_{i}(1),[G: A]=\lambda^{G}(1)=\sum_{i=1}^{u} \phi_{i}(1)^{2}$ $=\sum_{i=1}^{u} d_{i}^{2}$. However $[G: A]=\sum_{i=1}^{t} d_{i}^{2}$ and hence $u=t$ and $\phi_{1}, \ldots, \phi_{t}$ are inequivalent irreducible constituents of $\lambda^{G}$.

Let $G$ be a group with center $Z$. If $\chi$ is an irreducible character on $G$ such that $\chi(1)^{2}=[G: Z]$, then $\left.\chi\right|_{z}=\chi(1) \lambda$ and $\lambda^{G}=\chi(1) \chi$ for some linear character $\lambda$ on $Z$. Conversely, if $\lambda$ is a linear character on $Z$ such that $\lambda^{G}=\chi(1) \chi$ for some irreducible character $\chi$ on $G$, then $\chi(1)^{2}=[G: Z]$. Hence $G$ is a group of central type if and only if there is a linear character $\lambda$ on $Z$ such that $t=1$ where $t$ is the number in (1.6). Since $t$ is the number of conjugacy classes of $G / Z$ contained in $T(G, \lambda)$, then $t=1$ if and only if $T(G, \lambda)=Z$. These remarks verify the results in $\$ 1$ of [6]. Since for every linear character on $Z$ one can define a 2-cocycle on $G / Z$ as in the proof of Lemma 1.1, Theorem 1 of [3] follows from Lemma 1.1 and the above remarks.

There is another relationship which exists between the elements of the set $T(G, \lambda)$ and the irreducible constituents of $\lambda^{G}$ which will be useful later. 
(1.7) If $x \notin T(G, \lambda)$ and $\phi$ is any irreducible constituent of $\lambda^{G}$, then $\phi(x)=0$. If $x \in T(G, \lambda)$ then there is an irreducible constituent of $\lambda^{G}$ for which $\phi(x) \neq 0$.

Proof. Suppose $x \notin T(G, \lambda)$. Then there is an element $g \in G$, such that $g^{-1} x g=x a, a \in A, \lambda(a) \neq 1$. Then $\phi(x)=\phi\left(g^{-1} x g\right)=\phi(x a)=\lambda(a) \phi(x)$. Since $\lambda(a) \neq 1, \phi(x)=0$.

Let $\lambda^{G}=\sum_{i=1}^{i} \phi_{i}(1) \phi_{i}$ and let $T_{i}, 1 \leq i \leq t$, be inequivalent irreducible representations of $G, T_{i}$ corresponding to $\phi_{i}$. For each $i$ and each conjugacy class $C$ of $G, \sum_{x \in C} T_{i}(x)$ is a scalar matrix by Schur's lemma $[4,27.3, p$. 181]. Let $\Sigma_{x \in C} T_{i}(x)=k \cdot T_{i}(1), k \in K$. The trace of $k \cdot T_{i}(1)$ is $k \cdot \phi_{i}(1)$, and

$$
k \cdot \phi_{i}(1)=\sum_{x \in C} \operatorname{trace}\left(T_{i}(x)\right)=\sum_{x \in C} \phi_{i}(x)=n \cdot \phi_{i}\left(x_{0}\right),
$$

where $n$ is the number of elements in $C$ and $x_{0}$ is any element of $C$. Thus if $\phi_{i}\left(x_{0}\right)=0$ for any $x_{0} \in C$, then $\sum_{x \in C} T_{i}(x)$ is the zero matrix.

Let $T$ be a representation of $A$ corresponding to $\lambda$. Then for every $g \in G$, $T^{G}(g)$ is similar to $\bigoplus \sum_{i=1}^{t} \phi_{i}(1) T_{i}(g)$. Let $x \in T(G, \lambda)$ and suppose that $\phi_{i}(x)=0$ for all $1 \leq i \leq t$. If $C$ is the conjugacy class of $G$ containing $x$, then $\sum_{y \in C} T_{i}(y)$ is the zero matrix for every $i$, and hence $\sum_{y \in C} T^{G}(y)$ is the zero matrix. For all $g, h \in G$, there is $i, j$ so that $\left(\sum_{y \in C} T^{G}(y)\right)_{i j}=\sum_{y \in C} \dot{\lambda}\left(g^{-1} y h\right)$, and thus $\sum_{y \in C} \dot{\lambda}\left(g^{-1} y h\right)=0$ for all $g, h$ in $G$. In particular $\sum_{y \in C} \dot{\lambda}\left(x^{-1} y\right)=0$. If $x^{-1} y \in A$ for any $y \in C$, then $x^{-1} y \in x^{-1} \mathrm{Cl}(x) \cap A$. Since $x \in T(G, \lambda)$, $x^{-1} \mathrm{Cl}(x) \cap A \subseteq \operatorname{kernel}(\lambda)$. If $n$ is the number of $y$ 's in $C$ for which $x^{-1} y \in A$, then $\sum_{y \in C} \dot{\lambda}\left(x^{-1} y\right)=n \cdot 1=0$. Since $x \in C, n \geq 1$, contradicting the statement that $n=0$. Hence for some $i, \phi_{i}(x) \neq 0$.

We can summarize the results of this section in the following theorem.

Theorem 1.8. Let $\lambda$ be a linear character defined on a subgroup $A$ of the center of a finite group $G$. Let

$$
T(G, \lambda)=\left\{x \in G: x^{-1} g^{-1} x g \in \operatorname{kernel}(\lambda) \text { if } x^{-1} g^{-1} x g \in[G, G] \cap A\right\}
$$

and let $t$ be the number of conjugacy classes of $G / A$ contained in $T(G, \lambda) / A$. Then $\lambda^{G}$ has $t$ inequivalent irreducible constituents. If $\phi$ is an irreducible constituent of $\lambda^{G}$ and $x \notin T(G, \lambda)$, then $\phi(x)=0$. If $x \in T(G, \lambda)$, then there is an irreducible constituent $\phi$ of $\lambda^{G}$ for which $\phi(x) \neq 0$.

2. In this section we study groups which are not of central type but share properties with normal subgroups of index $p$ of groups of central type.

Definition. Let $H$ be a group with center $Z$. We call $H p$-special if there is a linear character $\lambda$ on $Z$, such that

(a) $\lambda^{H}$ has $p$ inequivalent irreducible constituents $\phi_{1}, \ldots, \phi_{p}$ all of the same degree, and

(b) if $\sum_{i=1}^{p} m_{i} \phi_{i}(x)=0$ for nonnegative integers $m_{i}$ and some $x \in H$, then either $\phi_{i}(x)=0$ for all $i$ or $m_{i}=m_{j}$ for all $i, j$.

We will also say $H$ is $p$-special with respect to $\lambda$. 
Note. Let $H$ be $p$-special with respect to $\lambda$ and $\lambda^{H}=e\left(\phi_{1}+\cdots+\phi_{p}\right)$. If $x \in H$, such that $\phi_{i}(x)=0$ for some $i$, then by condition (b), $\phi_{j}(x)=0$ for all j. If $T(H, \lambda)=\left\{x \in H: x^{-1} \mathrm{Cl}_{H}(x) \cap Z \subseteq \operatorname{kernel}(\lambda)\right\}$ then $\phi_{i}(x) \neq 0,1 \leq i$ $\leq p$, if and only if $x \in T(H, \lambda)$ by Theorem 1.8.

Theorem 2.1. If $G$ is a group of central type with center $Z(G)$ and $H$ is a normal subgroup of $G$ of index $p$, then $H$ is of central type if $Z(H) \neq Z(G)$ and $H$ is $p$ special if $Z(H)=Z(G)$.

Proof. Suppose $\chi$ is an irreducible character on $G$, so that $\chi(1)^{2}=[G: Z(G)]$. If $Z(G) \nsubseteq H$, then $\left.\chi\right|_{H}$ is irreducible since elements of $Z(G)$ are represented by scalar matrices by Schur's lemma. Hence $\left(\left.\chi\right|_{H}(1)\right)^{2}=\chi(1)^{2}=[G: Z(G)] \leq$ $[H: Z(H)]$. Therefore, $[Z(G): Z(H)]=p$ and $H$ is of central type. If $Z(G)$ $\subseteq H$, then $Z(G) \subseteq Z(H)$ and $[H: Z(H)]<[G: Z(G)]$. Hence $\left.\chi\right|_{H}$ cannot be irreducible. Since $H$ is a normal subgroup of $G$ of index $p$, by Clifford's theorem [4, Theorem 49.2, p. 343] either $\left.\chi\right|_{H}=p \phi$ where $\phi$ is irreducible on $H$ or $\left.\chi\right|_{H}=\phi_{1}+\cdots+\phi_{p}$ where $\phi_{1}, \ldots, \phi_{p}$ are conjugate irreducible characters on $H$. If $\left.\chi\right|_{H}=p \phi$, then, by Frobenius reciprocity [4, Theorem 38.8, p. 271], $\phi^{G}=p \chi+\cdots$ and $\phi^{G}(1)=p \phi(1) \geq p \chi(1)$ which is impossible. If $\left.\chi\right|_{H}=\phi_{1}$ $+\cdots+\phi_{p}$, then $H$ has an irreducible character of degree $\left([G: Z(G)] / p^{2}\right)^{1 / 2}$ and

$$
[H: Z(H)] \geq[G: Z(G)] / p^{2}=[H: Z(H)] \cdot[Z(H): Z(G)] / p .
$$

If $[Z(H): Z(G)]=p$, then $H$ has an irreducible character of degree $[H: Z(H)]$ and $H$ is of central type.

If $Z(H)=Z(G), H$ is not of central type. Let $Z=Z(G)=Z(H)$, and $\left.\chi\right|_{z}=\chi(1) \lambda$ where $\lambda$ is a linear character on $Z$. Then $\lambda^{H}=\phi_{1}(1) \phi_{1}+\cdots+$ $\phi_{p}(1) \phi_{p}$ and since the $\phi_{1}, \ldots, \phi_{p}$ are conjugate characters, they all have the same degree. Suppose $\sum_{i=1}^{p} m_{i} \phi_{i}(x)=0$ for nonnegative integers $m_{i}$ and some $x \in H$. If $x \notin T(H, \lambda)$ then, by Theorem $1.8, \phi_{i}(x)=0$ for every $i$. If $x \in Z$ then $\phi_{i}(x)=\phi_{i}(1) \lambda(x)$ and $0=\sum_{i=1}^{p} m_{i} \phi_{i}(x)=\sum_{i=1}^{p} m_{i} \phi_{1}(1) \lambda(x)$. Hence $\sum_{i=1}^{p} m_{i}$ $=0$ or $m_{i}=0$ for each $i$. Now suppose $x \in T(H, \lambda), x \notin Z$. Since $G$ is of central type, $T(G, \lambda)=Z$ and since $x \notin Z$, there is an element $g \in G$ such that $x^{-1} g^{-1} x g=z$ and $\lambda(z) \neq 1$. Since $x \in T(H, \lambda), g \notin H$ and by relabeling if necessary, we can assume that $\phi_{i}=\phi_{p}^{g^{i}}$ and $\phi_{p}(x) \neq 0$. Since $g^{p} \in H$, and $x^{-1} g^{-p} x g^{p}=z^{p}, \lambda\left(z^{p}\right)=1$, and $\lambda\left(z^{i}\right)$ is a primitive $p$ th root of 1 if $i \neq 0$ (modulo $p$ ). Then

$$
\begin{aligned}
0 & =\sum_{i=1}^{p} m_{i} \phi_{i}(x) \\
& =\sum_{i=1}^{p} m_{i} \phi_{p}\left(g^{-i} x g^{i}\right) \\
& =\sum_{i=1}^{p} m_{i} \lambda\left(x^{-1} g^{-i} x g^{i}\right) \phi_{p}(x) \\
& =\left(\sum_{i=1}^{p} m_{i} \lambda\left(z^{i}\right)\right) \phi_{p}(x) .
\end{aligned}
$$


Since $\phi_{p}(x) \neq 0, \sum_{i=1}^{p} m_{i} \lambda\left(z^{i}\right)=0$ and since $\lambda\left(z^{i}\right), 0 \leq i \leq p-1$, are $p$ distinct $p$ th roots of $1, m_{i}=m_{j}$ for all $i$ and $j$. This completes the proof of Theorem 2.1.

Note. If $H$ is a $p$-special group and $H$ is a normal subgroup of index $p$ in a group of central type and $x \in T(H, \lambda)$ then there is an element $g \in G$, so that $x^{-1} g^{-1} x g=z \in Z$ and $\lambda(z) \neq 1$. If we define an automorphism $\sigma$ on $H$ by $\sigma(h)=g^{-1} h g$, then

(a) $\sigma^{p}$ is an inner automorphism of $H$;

(b) $\sigma(z)=z$ for all $z \in Z$;

(c) $\sigma(x)=x \cdot z$ where $z \in Z, \lambda(z) \neq 1$ for some $x \in T(H, \lambda)$.

Thus if a $p$-special group $H$ is a normal subgroup of index $p$ of a group of central type, then there must be an automorphism of $H$ satisfying conditions (a), (b), and (c). We next show these conditions are sufficient.

Theorem 2.2. Let $H$ be a finite group with center $Z$ and let $\lambda$ be a linear character on $Z$ such that $H$ is p-special with respect to $\lambda$. Suppose there is an automorphism $\sigma$ of $H$, so that

(a) $\sigma^{p}$ is an inner automorphism of $H$;

(b) $\sigma(z)=z$ for all $z \in Z$;

(c) $\sigma(x)=x \cdot z$ where $z \in Z, \lambda(z) \neq 1$ for some $x \in T(H, \lambda)$ where $T(H, \lambda)$ $=\left\{x \in H: x^{-1} h^{-1} x h \in Z\right.$ iff $\left.x^{-1} h^{-1} x h \in \operatorname{kernel}(\lambda)\right\}$.

Then $H$ is a normal subgroup of index $p$ of a group of central type.

Proof. Let $G$ be the group generated by elements $h \in H$ and an element $g$ where $h g^{n}=g^{n} \sigma^{n}(h)$ for any integer $n$. Then $Z(G)=Z$ and since $\sigma^{p}$ is an inner automorphism of $H, H$ is a normal subgroup of index $p$ of $G$.

Since $H$ is $p$-special with respect to $\lambda, \lambda^{H}=e\left(\phi_{1}+\cdots+\phi_{p}\right)$ where the $\phi_{i}$ 's are inequivalent irreducible characters on $H$ and $\phi_{i}(1)=e$ for all $i$. Let $\chi$ be an irreducible constituent of $\lambda^{G}$. By Theorem 1.8, $\chi(y) \neq 0$ only if $y \in T(G, \lambda)$. If $x$ is the element given in part (c) of Theorem 2.2, $x \notin T(G, \lambda)$ and hence $\chi(x)=0$. Since $\chi$ is a constituent of $\lambda^{G},\left.\chi\right|_{H}=\sum_{i=1}^{p} m_{i} \phi_{i}$ where the $m_{i}$ 's are nonnegative integers. Then $\chi(x)=0=\sum_{i=1}^{p} m_{i} \phi_{i}(x)$. Since $H$ is $p$-special and $x \in T(H, \lambda)$, $m_{i}=m_{p}$ for all $i$. Hence $\left.\chi\right|_{H}=m_{p} \sum_{i=1}^{p} \phi_{i}$ and $\chi(1)=m_{p} \cdot p \cdot e$ or

$$
\begin{aligned}
\chi(1)^{2} & =m_{p}^{2} \cdot p^{2} \cdot e^{2}=m_{p}^{2} \cdot p^{2} \cdot[H: Z] / p \\
& =m_{p}^{2} \cdot p \cdot[H: Z]=m_{p}^{2} \cdot[G: Z]
\end{aligned}
$$

Since $\chi(1)^{2} \leq[G: Z], m_{p}=1, \chi(1)^{2}=[G: Z]$ and $G$ is a group of central type.

Example 2.3. Let $H=\left\langle x, y \mid x^{8}=y^{2}=1, y^{-1} x y=x^{-1}\right\rangle ; H$ is the dihedral group of order 16 and $H$ is 2-special. $Z(H)=\left\{1, x^{4}\right\}$. Let $\lambda$ be defined on $Z(H)$ by $\lambda\left(x^{4}\right)=-1$. If $\omega$ is a primitive 8th root of 1 , define $\sigma_{i}(x)=\omega^{i}$ for $0 \leq i \leq 7$. Let $X=\langle x\rangle$. Let $\left.\phi_{1}\right|_{x}=\sigma_{1}+\sigma_{7}$ with $\phi_{1}(h)=0$ for all $h \notin X$ and let $\left.\phi_{2}\right|_{x}$ $=\sigma_{3}+\sigma_{5}$ with $\phi_{2}(h)=0$ for all $h \notin X$. Then $\phi_{1}$ and $\phi_{2}$ are inequivalent 
irreducible characters on $H$ and are constituents of $\lambda^{H} . T(H, \lambda)=\left\{1, x, x^{3}, x^{4}, x^{5}\right.$, $\left.x^{7}\right\}$. If we define $\sigma$ by $\sigma(x)=x^{5}$ and $\sigma(y)=y$, then $\sigma$ satisfies the hypothesis of Theorem 2.2.

Example 2.4. Let $p$ be any prime, and let $H=\langle a, b, c, d, e| a^{p}=b^{p}=c^{p}$ $\left.=d^{p}=e^{p}=1, b^{-1} a b=a d, c^{-1} a c=a, c^{-1} b c=b e, d \in Z(H), e \in Z(H)\right\rangle$. Then $Z(H)=\langle d, e\rangle$. Let $\lambda$ be a linear character on $Z(H)$, defined by $\lambda(d)=\omega$, $\lambda(e)=1$, where $\omega$ is a primitive $p$ th root of 1 . Let $C=\langle c, d, e\rangle$. Let $\sigma_{i}\left(c^{s} \cdot z\right)$ $=\omega^{i s} \lambda(z)$. Then $\lambda^{c}=\sum_{i=1}^{p} \sigma_{i}$. Define $\phi_{i}$ on $H$ by $\left.\phi_{i}\right|_{c}=p \sigma_{i}$ and $\phi_{i}(h)=0$ for all $h \notin C, 1 \leq i \leq p$. Then $\phi_{1}, \ldots, \phi_{p}$ are inequivalent irreducible constituents of $\lambda^{H}$ and $H$ is $p$-special with respect to $\lambda$. $T(H, \lambda)=\langle c, d, e\rangle$. If $\sigma$ is defined by $\sigma(a)=a, \sigma(b)=b, \sigma(c)=c d, \sigma(d)=d, \sigma(e)=e$, then $\sigma$ satisfies the hypothesis of Theorem 2.2.

Example 2.5. Let $p$ be any prime, $p \neq 2$ and let $H=\langle x, y, u, v, z| x^{p^{2}}=z^{p^{2}}$ $=y^{p}=u^{p}=v^{p}=1, z \in Z(H), y^{-1} x y=x^{p+1}, u^{-1} x u=x y z, u^{-1} y u=y z^{p}$, $\left.v^{-1} x v=x, v^{-1} y v=y, v^{-1} u v=u z^{p}\right\rangle$. The center of $H$ is $\langle z\rangle$. Let $\lambda$ be a faithful linear character on $Z(H)$. Let $X=\langle x\rangle \cdot\langle z\rangle$ and let $\omega$ be a primitive $p^{2}$ root of 1. If $\sigma_{i}\left(x^{s} \cdot z\right)=\omega^{s i} \lambda(z)$, then $\lambda^{X}=\sum_{i=1}^{p^{2}} \sigma_{i}$. Define $\phi_{i}$ on $H$ by

$$
\left.\phi_{i}\right|_{x}=p \sigma_{p i}+\sum_{u=1}^{p} \sum_{v=1}^{p-1} \sigma_{p u+v}
$$

and $\phi_{i}(h)=0$ for all $h \notin X, 1 \leq i \leq p$. Then $\phi_{0}, \ldots, \phi_{p-1}$ are inequivalent irreducible constituents of $\lambda^{H}$, and $H$ is p-special with respect to $\lambda$. $T(H, \lambda)$ $=\left\{x^{s} z^{i}: 0 \leq i \leq p^{2}-1, s\right.$ relatively prime to $\left.p\right\}$. If $\sigma$ is defined by $\sigma(x)=x \cdot z^{p}$, $\sigma(y)=y, \sigma(u)=u, \sigma(v)=v, \sigma(z)=z$, then $\sigma$ satisfies the hypothesis of Theorem 2.2.

We will study p-special groups by studying the set $T(H, \lambda)$.

Lemma 2.6. Let $H$ be a p-special group with respect to $\lambda$ on the center $Z$, and suppose $[H, H] \cap$ kernel $(\lambda)=\{1\}$. Let $x \in T(H, \lambda), x \notin Z$ and $n$ be the minimum number so that $x^{n} \in Z$. Then $y \in T(H, \lambda)$ if and only if either $y \in Z$ or $y$ is conjugate to $x^{s} \cdot z$ for some s relatively prime to $n$ and some $z \in Z$.

Proof. If $x \in H$, let $\langle x\rangle$ denote the subgroup of $H$ generated by $x$. Let $x \in T(H, \lambda), x \notin Z, X=\langle x\rangle \cdot Z$ and $n=[X: Z]$. Let $\lambda^{H}=e\left(\phi_{1}+\cdots+\phi_{p}\right)$, $\lambda^{X}=\sigma_{1}+\cdots+\sigma_{n}$ and $\sigma_{i}^{H}=\sum_{j=1}^{p} k_{j i} \phi_{j}$.

Suppose $y \in T(H, \lambda), y \notin Z$, and $y$ is not conjugate to any element of $X$. Then

$$
\sigma_{i}^{H}(y)=0=\sum_{j=1}^{p} k_{j i} \phi_{j}(y) .
$$

Since $y \in T(H, \lambda)$ and $H$ is $p$-special, $k_{j i}=k_{p i}$ for all $j$. Hence $\sigma_{i}^{H}=k_{p i} \sum_{j-1}^{p} \phi_{j}$ and $\sigma_{i}^{H}(1)=[H: X]=k_{p i} \cdot p \cdot e$. Hence $k_{p i}=k_{p p}$ for all $i$ and $\left.\phi_{j}\right|_{X}$ $=\sum_{i=1}^{n} k_{j i} \sigma_{i}=k_{p p} \sum_{i=1}^{n} \sigma_{i}=k_{p p} \lambda^{X}$. Hence $\phi_{j}(x)=0$ for all $1 \leq j \leq p$ and $x \notin T(H, \lambda)$ by Theorem 1.8, which contradicts our choice of $x$. Thus if $y \in T(H, \lambda), y \notin Z$, then $y$ is conjugate to some element of $X$. 
Suppose $x^{s} \in T(H, \lambda)$ and $s$ and $n$ are not relatively prime. By the same argument as in the preceding paragraph, $x$ is conjugage to some element of $\left\langle x^{s}\right\rangle \cdot Z$. However, this is impossible since $\left[\left\langle x^{s}\right\rangle \cdot Z: Z\right]\langle[\langle x\rangle \cdot Z$ : $Z]$. Therefore $x^{s} \in T(H, \lambda)$ only if $s$ and $n$ are relatively prime.

Suppose $s$ and $n$ are relatively prime and $x^{s} \notin T(H, \lambda)$. Then there is an element $h \in H$ such that $x^{-s} h^{-1} x^{s} h=z \in Z$ and $z \neq 1$. Since $s$ and $n$ are relatively prime, there is an integer $t$ so that $s t \equiv 1$ (modulo $n$ ) and $x^{s t}=x \cdot z_{1}$ for some $z_{1} \in Z$. Since $x^{-s} h^{-1} x^{s} h=z, z^{t}=\left(x^{-s} h^{-1} x^{s} h\right)^{t}=x^{-s t} h^{-1} x^{s t} h$ $=x^{-1} h^{-1} x h$. If $z^{t}=1$, then $z=x^{-s} h^{-1} x^{s} h=\left(x^{-1} h^{-1} x h\right)^{s}=1$, which contradicts the choice of $h$. Hence $x \notin T(H, \lambda)$, which contradicts our choice of $x$. Therefore if $s$ and $n$ are relatively prime, then $x^{s} \in T(H, \lambda)$. If $x^{s} \in T(H, \lambda)$ then $x^{s} \cdot z \in T(H, \lambda)$ for all $z \in Z$, and any conjugate of $x^{s} \cdot z$ is an element of $T(H, \lambda)$.

Lemma 2.7. Let $H$ be a p-special group with respect to $\lambda$ on the center $Z$ and assume $[H, H] \cap$ kernel $(\lambda)=\{1\}$. Then every element of $T(H, \lambda) / Z$ has order a power of $p$ in $H / Z$.

Proof. Let $\lambda^{H}=e\left(\phi_{1}+\cdots+\phi_{p}\right)$. Let $S_{p}$ be a $p$ Sylow subgroup of $H$, $R=S_{p} \cdot Z$ and let $\gamma$ be an irreducible constituent of $\lambda^{R}$. By Schur's lemma, elements of $Z$ are represented by scalar matrices, and hence $\gamma$ restricted to $S_{p}$ is irreducible and $\gamma(1)$ is a power of $p$. Since $\gamma^{H}$ is a constituent of $\lambda^{H}, \gamma^{H}$ $=\sum_{i=1}^{p} m_{i} \phi_{i}$ for some nonnegative integers $m_{i}$. Now

$$
\gamma^{H}(1)=[H: R] \gamma(1)=\sum_{i=1}^{p} m_{i} e
$$

and

$$
[H: R]^{2} \gamma(1)^{2}=\left(\sum_{i=1}^{p} m_{i}\right)^{2} e^{2}=\left(\sum_{i=1}^{p} m_{i}\right)^{2}[H: Z] / p
$$

By taking $p$-parts, we get the equation

$$
\gamma(1)^{2}=\left(\sum_{i=1}^{p} m_{i}\right)_{p}^{2}[R: Z] / p
$$

Since $\gamma(1)^{2} \leq[R: Z], \gamma(1)^{2}=[R: Z] / p$. Thus $\lambda^{R}=e_{p}\left(\gamma_{1}+\cdots+\gamma_{p}\right)$ where $\gamma_{1}, \ldots, \gamma_{p}$ are inequivalent irreducible characters on $R$ and $\gamma_{i}(1)^{2}=e_{p}^{2}=$ $[R: Z] / p$ for all $i$.

Suppose $x \in T(H, \lambda)$ and $x$ is not conjugate to any element of $R$. Then $\gamma_{i}^{H}(x)=0$ for all $i$. Let $\gamma_{i}^{H}=\sum_{j=1}^{p} k_{i j} \phi_{j}$. Then

$$
\gamma_{i}^{H}(x)=0=\sum_{j=1}^{p} k_{i j} \phi_{j}(x)
$$


Since $x \in T(H, \lambda), \phi_{j}(x) \neq 0$ for some $j$ and since $H$ is $p$-special, $k_{i j}=k_{i p}$ for all $j$. Hence

$$
\gamma_{i}^{H}=k_{i p}\left(\phi_{1}+\cdots+\phi_{p}\right) \text { and } \gamma_{i}^{H}(1)=[H: R] \gamma_{i}(1)=k_{i p} \cdot p \cdot e .
$$

By taking $p$-parts, we have $e_{p}=\gamma_{i}(1)=\left(k_{i p}\right)_{p} \cdot p \cdot e_{p}$ which is clearly impossible. Thus if $x \in T(H, \lambda), x$ is conjugate to an element of $R$. Since $R=S_{p} \cdot Z$, the order of $x Z$ in $H / Z$ is a power of $p$.

Theorem 2.8. Let $H$ be a group with center $Z$, and let $\lambda$ be a linear character on Z. Let

$$
T(H, \lambda)=\left\{x \in H: x^{-1} \mathrm{Cl}_{H}(x) \cap Z \subseteq \operatorname{kernel}(\lambda)\right\} .
$$

If $H$ is p-special with respect to $\lambda$ then for any $p$ Sylow subgroup $S$ of $H$, there is an $x \in S$ such that

(a) $T(R, \lambda) \cup$ 作 $\mathrm{Cl}_{R}\left(x^{i}\right) \cdot Z$ where $R=S \cdot Z$;

(b) $T(H, \lambda)=\bigcup_{i=0}^{p-1} \mathrm{Cl}_{H}\left(x^{i}\right) \cdot Z$;

(c) for $i \neq \equiv$ (modulo $p$ ), $\mathrm{Cl}_{H}\left(x^{i}\right) \cdot Z=\mathrm{Cl}_{H}\left(x^{j}\right) \cdot Z$ if and only if $i \equiv j$ (modulo p).

Proof. We prove the theorem first in the case that $[H, H] \cap \operatorname{kernel}(\lambda)=\{1\}$. Let $S$ be a $p$ Sylow subgroup and let $R=S \cdot Z$. As in the proof of Lemma 2.7, $\lambda^{R}$ has $p$ inequivalent irreducible constituents. By Theorem 1.8, $T(R, \lambda) / Z$ consists of $p$ distinct conjugacy classes of $R / Z$. Let $x \in T(R, \lambda), x \notin Z$. Since $R=S \cdot Z$, we can choose $x \in S \cap T(R, \lambda)$. As in the proof of Lemma 2.6, $\mathrm{Cl}_{R}\left(x^{i}\right) \cdot Z \subseteq T(R, \lambda)$ for all $i$ relatively prime to $p$. Since $R / Z$ is a $p$ group, $x^{r} \cdot Z$ and $x^{s} \cdot Z$ are conjugate in $R / Z$ only if $r \equiv s$ (modulo $p$ ). Since $T(R, \lambda) / Z$ consists of exactly $p$ distinct conjugacy classes of $H / Z$,

$$
T(R, \lambda)=\bigcup_{i=0}^{p-1} \mathrm{Cl}_{r}\left(x^{i}\right) \cdot Z .
$$

Moreover, for $i \not \equiv 0$ (modulo $p$ ), $\mathrm{Cl}_{R}\left(x^{i}\right) \cdot Z=\mathrm{Cl}_{R}\left(x^{j}\right) \cdot Z$ if and only if $i \equiv j$ (modulo $p$ ).

Let $y \in T(H, \lambda), y \notin Z$. By Lemma $2.7, y Z$ is a $p$ element in $H / Z$, and since all $p$ Sylow subgroups of $H$ are conjugates, $\mathrm{Cl}_{H}(y) \cap R \neq \varnothing$. Let $y^{\prime} \in \mathrm{Cl}_{H}(y)$ $\cap R$. Then $y^{\prime} \in T(H, \lambda) \cap R$ and $y^{\prime} \in T(R, \lambda)$. Then $y^{\prime} \in \mathrm{Cl}_{R}\left(x^{i}\right) \cdot Z$ for some $1 \leq i \leq p-1$. Hence $y^{\prime}=r^{-1} x^{i} r \cdot z$ for some $r \in R, z \in Z$, and $\mathrm{Cl}_{H}\left(y^{\prime}\right) \cdot Z$ $=\mathrm{Cl}_{H}\left(x^{i}\right) \cdot Z$. But $y \in \mathrm{Cl}_{H}\left(y^{\prime}\right)$ and hence $y \in \mathrm{Cl}_{H}\left(x^{i}\right) \cdot Z$. Thus for every $y \in T(h, \lambda), y \notin Z, y \in \mathrm{Cl}_{H}\left(x^{i}\right) \cdot Z$ for some $i$. Therefore

$$
T(H, \lambda) \subseteq \bigcup_{i=0}^{p-1} \mathrm{Cl}_{H}\left(x^{i}\right) \cdot Z
$$

Since $\lambda^{H}$ has $p$ inequivalent irreducible constituents, by Theorem $1.8, T(H, \lambda) / Z$ consists of exactly $p$ distinct conjugacy classes of $H / Z$. Therefore 


$$
T(H, \lambda)=\bigcup_{i=0}^{p-1} \mathrm{Cl}_{H}\left(x^{i}\right) \cdot Z
$$

and

$$
\mathrm{Cl}_{H}\left(x^{i}\right) \cdot Z \neq \mathrm{Cl}_{H}\left(x^{j}\right) \cdot Z
$$

for $i \neq j, 0 \leq i, j \leq p-1$. Since for $i \neq 0$ (modulo $p$ ), $\mathrm{Cl}_{R}\left(x^{i}\right) \cdot Z=\mathrm{Cl}_{R}\left(x^{j}\right)$ - $Z$ if $i \equiv j$ (modulo $p$ ), then $\mathrm{Cl}_{H}\left(x^{i}\right) \cdot Z=\mathrm{Cl}_{H}\left(x^{j}\right) \cdot Z$ for $i \equiv j$ (modulo $p$ ), $i \not \equiv 0$ (modulo $p$ ). Hence, for $i \not \equiv 0$ (modulo $p$ ), $\mathrm{Cl}_{H}\left(x^{i}\right) \cdot Z=\mathrm{Cl}_{H}\left(x^{j}\right) \cdot Z$ if and only if $i \equiv j$ (modulo $p$ ). This completes the proof of Theorem 2.8 in the case that $\lambda$ is faithful on $[H, H] \cap Z$.

If $\lambda$ is not faithful on $[H, H] \cap Z$, let $N=[H, H] \cap \operatorname{kernel}(\lambda)$. Let $\bar{H}=H / N$, $\bar{Z}=Z / N$, and $\bar{\lambda}$ be a linear character on $\bar{Z}$ defined by $\bar{\lambda}(z N)=\lambda(z)$ for any $z \in z N$. If $\lambda^{H}=e\left(\phi_{1}+\cdots+\phi_{p}\right)$, define $\bar{\phi}_{i}(h N)=\phi_{i}(h)$ for any $h \in h N$. Then $\bar{\phi}_{1}, \ldots, \bar{\phi}_{p}$ are inequivalent irreducible constituents of $\bar{\lambda}^{H}$, each of degree $e$. Let $\bar{S}$ be any $p$ Sylow subgroup of $\bar{H}$, and let $\bar{R}=\bar{S} \cdot \bar{Z}$.

If $Z(\bar{H}) \neq \bar{Z}$, let

$$
\left.\bar{\phi}_{i}\right|_{Z(H)}=\bar{\phi}_{i}(1) \sigma_{i}=e \sigma_{i}
$$

where $\sigma_{i}$ is a linear character on $Z(\bar{H})$. Then

$$
\begin{aligned}
\left.\bar{\lambda}^{H}\right|_{z(H)} & =[\bar{H}: Z(\bar{H})] \bar{\lambda}^{z(H)} \\
& =e\left(\left.\bar{\phi}_{1}\right|_{z(H)}+\cdots+\left.\bar{\phi}_{p}\right|_{z(H)}\right) \\
& =e^{2}\left(\sigma_{1}+\cdots+\sigma_{p}\right)
\end{aligned}
$$

Hence

$$
\bar{\lambda}^{Z(H)}=\sigma_{1}+\cdots+\sigma_{p}, \quad[Z(\bar{H}): \bar{Z}]=p, \quad \text { and } e^{2}=[\bar{H}: Z(\bar{H})]
$$

Hence $\bar{H}$ is of central type. By Theorem 2 of [3], $S$ is of central type and $Z(\bar{S})=Z(\bar{H}) \cap \bar{S}$. Since $[Z(\bar{H}): \bar{Z}]=p$,

$$
[Z(\bar{S}): \bar{Z} \cap \bar{S}]=[Z(\bar{H}) \cap \bar{S}: \bar{Z} \cap \bar{S}]=p .
$$

Let $\bar{x} \in Z(\bar{S}), \bar{x} \notin \bar{Z}$. Since $\bar{S}$ is of central type, $\bar{R}=\bar{S} \cdot \bar{Z}$ is of central type and $Z(\bar{R})=Z(\bar{H})$. Now $Z(\bar{R}) \subseteq T(\bar{R}, \bar{\lambda})$ and since $T(\bar{R}, \bar{\lambda}) / \bar{Z}$ contains $p$ conjugacy classes of $\bar{R} / \bar{Z}$ by Theorem 1.8 and $[Z(\bar{R}): \bar{Z}]=p, Z(\bar{R})=T(\bar{R}, \bar{\lambda})$ and $T(\bar{R}, \bar{\lambda})=\bigcup_{i=0}^{p-1} \mathrm{Cl}_{\bar{R}}\left(\bar{x}^{i}\right) \cdot \bar{Z}$. Also $T(\bar{H}, \bar{\lambda})=Z(\bar{H})$ and $T(\bar{H}, \bar{\lambda})$ $=\bigcup_{i=0}^{p-1} \mathrm{Cl}_{H}\left(\bar{x}^{i}\right) \cdot \bar{Z}$. Moreover $\mathrm{Cl}_{H}\left(\bar{x}^{i}\right) \cdot \bar{Z}=\mathrm{Cl}_{H}\left(\bar{x}^{j}\right) \cdot \bar{Z}$ if and only if $i=j$ (modulo $p)$. 
If $Z(\bar{H})=\bar{Z}$ and $\sum_{i=1}^{p} m_{i} \bar{\phi}_{i}(x N)=0$ for some $x N \in \bar{H}$ and some nonnegative integers $m_{i}$, then $\sum_{i=1}^{p} m_{i} \phi_{i}(x)=0$ for some $x \in H$. Since $H$ is $p$-special, either $\phi_{i}(x)=0$ for all $i$, or $m_{i}=m_{j}$ for all $i, j$. Hence $\bar{H}$ is $p$-special. Since $\bar{\lambda}$ is faithful on $[\bar{H}, \bar{H}] \cap \bar{Z}$, for any $p$ Sylow subgroup $\bar{S}$ of $\bar{H}$, there is an $\bar{x} \in \bar{S}$ so that

(a) $T(\bar{R}, \bar{\lambda})=\bigcup_{i=0}^{p-1} \mathrm{Cl}_{\bar{R}}\left(\bar{x}^{i}\right) \cdot \bar{Z}$ where $\bar{R}=\bar{S} \cdot \bar{Z}$.

(b) $T(\bar{H}, \bar{\lambda})=\bigcup_{i=0}^{p-1} \mathrm{Cl}_{H}\left(\bar{x}^{i}\right) \cdot \bar{Z}$.

(c) For $i \neq 0$ (modulo $p$ ), $\mathrm{Cl}_{H}\left(\bar{x}^{-i}\right) \cdot \bar{Z}=\mathrm{Cl}_{H}\left(\bar{x}^{j}\right) \cdot \bar{Z}$ if and only if $i \equiv j$ (modulo $p$ ). Hence, regardless of whether $Z(\bar{H})=\bar{Z}$ or not, for any $p$ Sylow subgroup $\bar{S}$ of $\bar{H}$, there is an $\bar{x} \in \bar{S}$, so that conditions (a), (b), and (c) are satisfied.

Let $S$ be a $p$ Sylow subgroup of $H$. If $\bar{S}$ is the natural image of $S$ in $H$, then $\bar{S}$ is a $p$ Sylow subgroup of $\bar{H}$. Let $R=S \cdot Z$ and $\bar{R}=\bar{S} \cdot \bar{Z}$. Since $N \subseteq \operatorname{kernel}(\lambda)$, it can be easily verified that

$$
\overline{T(H, \lambda)}=T(\bar{H}, \bar{\lambda}) \text { and } \overline{T(R, \lambda)}=T(\bar{R}, \bar{\lambda}) \text {. }
$$

Let $x \in S$, such that $x N=\bar{x}$ and $\bar{X} \in \bar{S}$, satisfying conditions (a), (b), and (c). Then

(a) $T(R, \lambda)=\bigcup_{i=1}^{p-1} \mathrm{Cl}_{R}\left(x^{i}\right) \cdot Z$.

(b) $T(H, \lambda)=\bigcup_{i=0}^{p-1} \mathrm{Cl}_{H}\left(x^{i}\right) \cdot Z$.

(c) For $i \neq 0$ (modulo $p$ ), $\mathrm{Cl}_{H}\left(x^{i}\right) \cdot Z=\mathrm{Cl}_{H}\left(x^{j}\right) \cdot Z$. if and only if $i=j$ (modulo $p$ ).

This completes the proof of Theorem 2.8 .

Let $H$ be $p$-special with respect to $\lambda$ and let $\lambda^{H}=e\left(\phi_{1}+\cdots+\phi_{p}\right)$. In the previous proofs, the condition

(b) if $\sum_{i=1}^{p} m_{i} \phi_{i}(x)=0$ for some nonnegative integers $m_{i}$ and some $x \in H$, then either $\phi_{i}(x)=0$ for all $i$, or $m_{i}=m_{j}$ for all $i, j$ is used of ten. The following example shows that this condition is necessary in Theorem 2.5.

Example 2.9. Let $H=\langle x, y, w| x^{5}=y^{4}=w^{2}=1, w^{-1} y w=y^{3}, y^{-1} x y=x$, $\left.w^{-1} x w=x^{4}\right\rangle$. Then $Z(H)=\left\langle y^{2}\right\rangle$ and $[H: Z]=20$. Let $Y=\langle x y\rangle$, let $\omega_{0}$ be a 10 th root of -1 , and let $\omega$ be a primitive 10th root of 1 . Let $\sigma_{i}\left((x y)^{s}\right)=\omega_{0}^{s} \omega^{s t}$. If $\lambda$ is defined on $Z(H)$ by $\lambda\left(y^{2}\right)=-1$, then $\lambda^{Y}=\sum_{i=0}^{g} \sigma_{i}$. Let $\phi_{1}, \ldots, \phi_{5}$ be defined on $H$ by the following: $\left.\phi_{1}\right|_{Y}=\sigma_{1}+\sigma_{4},\left.\phi_{2}\right|_{Y}=\sigma_{2}+\sigma_{3},\left.\phi_{3}\right|_{Y}=\sigma_{7}+\sigma_{8}$, $\left.\phi_{4}\right|_{Y}=\sigma_{6}+\sigma_{9},\left.\phi_{5}\right|_{Y}=\sigma_{0}+\sigma_{5}$, and $\phi_{i}(h)=0$ for all $h \notin Y$ and $1 \leq i \leq 5$. Then $\phi_{1}, \ldots, \phi_{5}$ are inequivalent irreducible characters on $H$, each of degree 2 , and $\lambda^{H}=2\left(\phi_{1}+\cdots+\phi_{5}\right)$. Notice that $\phi_{5}\left(x^{s} y\right)=0$ while $\phi_{i}\left(x^{s} y\right) \neq 0$ for $i \neq 5$. Hence condition (b) is not satisfied.

Throughout the remainder of this section we will be working toward a converse of Theorem 2.8. We will need the following algebraic facts [4, Example 1, p. 13]:

(2.10) If $p$ is a prime, $p \neq 2$, and $\alpha$ is a positive integer, then

(a) $(p+1)^{p^{a-1}}=a p^{\alpha}+1$ where $a \equiv 1$ (modulo $p$ ).

(b) for every $0 \leq a \leq p^{\alpha-1}-1$, there is a unique $0 \leq t \leq p^{\alpha-1}-1$ so that $(p+1)^{t} \equiv a p+1\left(\right.$ modulo $\left.p^{\alpha}\right)$. 
Lemma 2.11. If $H$ is a group with center $Z$ and

$$
T(H)=\left\{x \in H: x^{-1} \mathrm{Cl}(x) \cap Z=\{1\}\right\}=\bigcup_{i=0}^{p-1} \mathrm{Cl}\left(x^{i}\right) \cdot Z
$$

for some $x$ which has order a power of $p$, then for all $1 \leq i \leq p-1$ and all positive integers $a$

$$
\mathrm{Cl}\left(x^{(a p+1) i}\right) \cdot Z=\mathrm{Cl}\left(x^{i}\right) \cdot Z
$$

Proof. Let $s$ be any number relatively prime to $p$. If $x^{s} \notin T(H)$ then there is an element $h \in H$, so that $h^{-1} x^{s} h=x^{s} \cdot z$ where $z \neq 1$. Since $s$ is relatively prime to $p$, there is an integer $a$ so that $x^{a s}=x$, and

$$
h^{-1} x^{a s} h=h^{-1} x h=x \cdot z^{a} .
$$

Since $z \neq 1, z^{a} \neq 1$. But this implies that $x \notin T(H)$, which is a contradiction. Therefore $x^{s} \in T(H)$ for all $s$ relatively prime to $p$.

Assume $p \neq 2$. Let $\alpha$ be the minimum number so that $x^{p^{a}} \in Z$. Let $A$ be the multiplicative group of integers, modulo $p^{\alpha}$. Let $A_{1}=\left\{a \in A: x \in \mathrm{Cl}\left(x^{a}\right) \cdot Z\right.$. Then

(2.12) $A_{1}$ is a subgroup of $A$ and $\left[A: A_{1}\right]$ divides $p-1$.

Proof. Suppose $a, b \in A_{1}$. Then there are $h_{1}, h_{2} \in H, z_{1}, z_{2} \in Z$ that $x=h_{1}^{-1} x^{a} h_{1} z_{1}$ and $x=h_{2}^{-1} x^{b} h_{2} z_{2}$. Then

$$
\begin{aligned}
x^{a^{-1} b} & =\left(h_{1}^{-1} x h_{1} z_{1}^{a^{-1}}\right)^{b}=h_{1}^{-1} x^{b} h_{1} z_{1}^{a^{-1} b} \\
& =h_{1}^{-1}\left(h_{2} x h_{2}^{-1} z_{2}^{-1}\right) h_{1} z_{1}^{a^{-1} b} \\
& =\left(h_{2}^{-1} h_{1}\right)^{-1} \times h_{2}^{-1} h_{1} z_{2}^{-1} z_{1}^{a^{-1} b}
\end{aligned}
$$

or

$$
x=h_{2}^{-1} h_{1} x^{a^{-1} b}\left(h_{2}^{-1} h_{1}\right)^{-1} z_{2} z_{1}^{-a^{-1} b} .
$$

Thus $a^{-1} b \in A_{1}$ and $A_{1}$ is a subgroup of $A$.

If $A_{i}=\left\{a \in A: x^{i} \in \mathrm{Cl}\left(x^{a}\right) \cdot Z\right\}$, then the mapping $a \rightarrow a i$ is a one-to-one mapping of $A_{1}$ onto $A_{i}$, for $1 \leq i \leq p-1$. For every $a \in A, x^{a} \in T(H)$ and $x^{a} \notin Z$. Therefore

$$
x^{a} \in \bigcup_{i=1}^{p-1} \mathrm{Cl}\left(x^{i}\right) \cdot Z, \quad A=\bigcup_{i=1}^{p-1} A_{i}, \quad \text { and }[A: 1] \leq(p-1)\left[A_{1}: 1\right] .
$$

Therefore $\left[A: A_{1}\right] \leq p-1$. Since $[A: 1]=(p-1) p^{\alpha-1},\left[A: A_{1}\right]$ divides $p-1$. This completes the proof of (2.12).

Since $\left[A: A_{1}\right]$ divides $p-1$, for every $a \in A, a^{p-1} \in A_{1}$. Since

$$
\left\{(p+1)^{t(p-1)}: 0 \leq t \leq p^{\alpha-1}-1\right\}=\left\{(p+1)^{t}: 0 \leq t \leq p^{\alpha-1}-1\right\},
$$


for every $t$, there is $t_{1}$ so that $(p+1)^{t}=(p+1)^{t_{1}(p-1)}$. Therefore $(p+1)^{t} \in A_{1}$ for $0 \leq t \leq p^{\alpha-1}-1$. By (2.10)(b) for every $0 \leq a \leq p^{\alpha-1}-1$, ap $+1 \in A_{1}$. Hence $x \in \mathrm{Cl}\left(x^{a p+1}\right) \cdot Z$ and $\mathrm{Cl}(x) \cdot Z=\mathrm{Cl}\left(x^{a p+1}\right) \cdot Z$. Since $(a p+1) \in A_{1}$, $(a p+1) i \in A_{i}$. Hence $x^{i} \in \mathrm{Cl}\left(x^{(a p+1) i}\right) \cdot Z$ or $\mathrm{Cl}\left(x^{i}\right) \cdot Z=\mathrm{Cl}\left(x^{(a p+1) i}\right) \cdot Z$.

If $p=2$, then $\mathrm{Cl}\left(x^{0}\right) \cdot Z \cap \mathrm{Cl}\left(x^{1}\right) \cdot Z=\varnothing$. For any $a$, since $x^{2 a+1} \in T(H)$, $x^{2 a+1} \in \mathrm{Cl}(x) \cdot Z$. Therefore

$$
\mathrm{Cl}(x) \cdot Z=\mathrm{Cl}\left(x^{2 a+1}\right) \cdot Z \text {. }
$$

We now prove the following crucial lemma:

Lemma 2.13. Let $H$ be a group with center $Z$ and assume $[H, H] \cap Z$ is cyclic. Let $\lambda$ be a linear character on $Z$, with $\lambda$ faithful on $[H, H] \cap Z$. Assume $\lambda^{H}=e\left(\phi_{1}+\cdots+\phi_{p}\right)$ where $\phi_{1}, \ldots, \phi_{p}$ are inequivalent irreducible characters on $H$ and $T(H, \lambda)=\bigcup_{i=0}^{p-1} \mathrm{Cl}\left(x^{i}\right) \cdot Z$ for some $x \in H$, where $x Z$ has order a power of p. Then $H$ is p-special.

Proof. By Schur's lemma, $\left.\phi_{i}\right|_{z}=\phi_{i}(1) \lambda$ and since $\left(\phi_{i}, \lambda^{H}\right)=e, \phi_{i}(1)=e$, $1 \leq i \leq p$. If $p=2$, assume $m_{1} \phi_{1}(y)+m_{2} \phi_{P}(y)=0$ for some $y \in H$ and nonnegative integers $m_{1}$ and $m_{2}$. If $y \notin T(H, \lambda)$, then $\phi_{i}(y)=0, i=1,2$, by Theorem 1.8. If $y \in Z$, then $\phi_{i}(y)=e \lambda(y)$ and $0=m_{1} \phi_{1}(y)+m_{2} \phi_{2}(y)=$ $e\left(m_{1}+m_{2}\right) \lambda(y)$. Thus $m_{1}=m_{2}=0$. If $y \in T(H, \lambda), y \notin Z$, then $\phi_{i}(y) \neq 0$ for $i=1$ or $i=2$. Since

$$
0=\lambda^{H}(y)=e\left(\phi_{1}(y)+\phi_{2}(y)\right)
$$

$\phi_{2}(y)=-\phi_{1}(y)$. Then

$$
0=m_{1} \phi_{1}(y)+m_{2} \phi_{2}(y)=\left(m_{1}-m_{2}\right) \phi_{1}(y) .
$$

Since $\phi_{1}(y) \neq 0, m_{1}=m_{2}$. Thus $H$ is $p$-special if $p=2$.

Assume $p \neq 2$. Let $\alpha$ be the minimum number so that $x^{p^{a}} \in Z$. Let $\omega_{0}$ be any $p^{\alpha}$ th root of $\lambda\left(x^{p^{\alpha}}\right)$ and let $\omega$ be a primitive $p^{\alpha}$ th root of 1 . Define $\sigma_{i}\left(x^{s} \cdot z\right)$ $=\omega_{0}^{s} \lambda(z) \cdot \omega^{i s}$. If $X=\langle x\rangle \cdot Z$, then $\sigma_{i}$ is independent of the way elements of $X$ are represented, and $\sigma_{i}, 0 \leq i \leq p^{\alpha}-1$, is a linear character on $X$. Since $\sigma_{i}(z)=\lambda(z)$ for all $z \in Z,\left(\sigma_{i}, \lambda^{X}\right)=1$. Hence

$$
\lambda^{X}=\sum_{v=0}^{p^{a}-1} \sigma_{v}
$$

We show the following:

(2.14) For a suitable $\omega_{0}$, there are integers $K$ and $k_{u, j}, 0 \leq u \leq p-1$, $1 \leq j \leq p$, such that

$$
\left.\phi_{j}\right|_{x}=\sum_{u=0}^{p-1} k_{u j} \sigma_{p^{\alpha-1} u_{u}}+K \sum_{\nu=0}^{p^{\alpha}-1} \sigma_{v}
$$


Moreover, $K=e / p^{\alpha}$ if $\alpha \neq 1$.

Proof. If $\alpha=1$, then $\lambda^{x}=\sigma_{0}+\cdots+\sigma_{p-1}$. Since

$$
\left.\lambda^{H}\right|_{X}=[H: X] \lambda^{X}=e\left(\left.\sum_{j=1}^{p} \phi_{j}\right|_{X}\right)=[H: X]\left(\sum_{i=0}^{p-1} \sigma_{i}\right)
$$

then $\left.\phi_{j}\right|_{x}=\sum_{u=0}^{p-1} k_{u j} \sigma_{u}$ for integers $k_{u j}$. If $K=0$, then (2.14) follows if $\alpha=1$.

If $\alpha \geq 2$, by Lemma 2.11, $x^{p+1} \in \mathrm{Cl}(x) \cdot Z$. Hence there is $h \in H, z_{0} \in Z$, so that

$$
h^{-1} x h=x^{p+1} z_{0}
$$

We wish to compute $h^{-t} x h^{t}$.

(2.16) If $h^{-1} x h=x^{\beta} \cdot z$ for any integer $\beta$ and $z \in Z$, then

$$
h^{-t} x h^{t}=x^{\beta^{t}} z^{\alpha(t)}
$$

where $e(t)=\left(\beta^{t}-1\right) /(\beta-1)$.

Proof. If $t=1$, the assertion follows by hypothesis. Suppose the assertion is true for $t=k$. Then

$$
\begin{aligned}
h^{-(k+1)} x h^{(k+1)} & =h^{-1} h^{-k} x h^{k} h \\
& =h^{-1} x^{\beta^{k}} h z^{e(k)}=\left(h^{-1} x h\right)^{\beta^{k}} z^{e(k)} \\
& =\left(x^{\beta} z\right)^{\beta^{k}} z^{e(k)}=x^{\beta^{k+1}} z^{\beta^{k}+e(k)}
\end{aligned}
$$

and $\beta^{k}+e(k)=\beta^{k}+\left(\beta^{k}-1\right) /(\beta-1)=\left(\beta^{k+1}-1\right) /(\beta-1)=e(k+1)$. By induction the assertion holds for all positive integers $t$.

By (2.10), $(p+1)^{p^{a-1}}=a p^{\alpha}+1$ where $a \equiv 1$ (modulo $p$ ). From equation (2.15) we get

$$
\begin{aligned}
h^{-p^{a-1}} x h^{p^{a-1}} & =x^{(p+1) p^{p-1}} z_{0}^{a(p a-1)} \\
& =x^{a p^{a}+1} \cdot z_{0}^{a p-1} \\
& =x\left(x^{p^{a}} z f^{p^{a-1}}\right)^{a}
\end{aligned}
$$

Since $x \in T(H, \lambda),\left(x^{p^{*}} z f^{p-1}\right)^{a}=1$. Since $a \equiv 1$ (modulo $p$ ), $x^{p^{*}} z p^{a-1}=1$. Choose $\omega_{0}$ such that $\lambda\left(z_{0}\right)=\omega_{0}^{-p}$.

If $s \neq 0$ (modulo $p$ ) and $g^{-1} x^{s} g \in X$, then $g^{-1} x g \in X$ and $g^{-1} x g=x^{i} z$ for some $z \in Z$. Since $\lambda^{H}$ has $p$ inequivalent irreducible constituents, by Theorem 1.8, $T(H, \lambda) / Z$ consists of $p$ distinct conjugacy classes of $H / Z$. Since

$$
T(H, \lambda)=\bigcup_{i=0}^{p-1} \mathrm{Cl}\left(x^{i}\right) \cdot Z,
$$


$\mathrm{Cl}\left(x^{\prime}\right) \cdot Z \neq \mathrm{Cl}\left(x^{j}\right) \cdot Z$ if $i \neq j, 0 \leq i, j \leq p-1$. Thus $\mathrm{Cl}\left(x^{i}\right) \cdot Z \cap \mathrm{Cl}\left(x^{j}\right) \cdot Z$ $=\varnothing$ if $i \neq j, 0 \leq i, j \leq p-1$. Since $g^{-1} x g=x^{i} z, i \equiv 1$ (modulo $p$ ) by Lemma 2.11. Then $i=a p+1$ for some $0 \leq a \leq p^{\alpha-1}-1$ and by (2.10) there is an integer $t$ such that

$$
i \equiv(p+1)^{\ell} \quad\left(\operatorname{modulo} p^{\alpha}\right)
$$

By (2.15) and (2.16), $h^{-t} x h^{t}=x^{(p+1)^{t}} \cdot z_{0}^{(t)}$ and $g^{-1} x g=x^{i} z=x^{(p+1)^{t}} \cdot z^{\prime}$ $=h^{-t} x h^{t} z_{0}^{-e(t)} \cdot z^{\prime}$, for some $z^{\prime} \in Z$. Hence

$$
h^{t} g^{-1} x g h^{-t}=x z_{0}^{-e(t)} \cdot z^{\prime}
$$

Since $x \in T(H, \lambda), z_{0}^{-e(t)} z^{\prime}=1$ and

$$
g h^{-t} \in C=\{h \in H: h x=x h\} .
$$

Thus if $g^{-1} x^{s} g \in X$ for any $s \neq 0$ (modulo $p$ ) then $g=c h^{t}$ for some $c \in C$ and $0 \leq t \leq p^{\alpha-1}-1$. Then for $s \neq 0($ modulo $p)$

$$
\begin{aligned}
\sigma_{i}^{H}\left(x^{s}\right) & =(1 /[X: 1]) \sum_{s \in H} \dot{\sigma}_{i}\left(g^{-1} x^{s} g\right) \\
& =(1 /[X: 1]) \sum_{c \in C} \sum_{i=0}^{p a-1-1} \sigma_{i}\left(h^{-t} c^{-1} x^{s} c h^{t}\right) \\
& =[C: X] \sum_{i=0}^{p a-1-1} \sigma_{i}\left(x^{(p+1)^{t} s} \cdot z_{0}^{(t) s}\right) \\
& =[C: X] \sum_{t=0}^{p a-1-1} \omega_{0}^{(p+1)^{\prime} \cdot s} \omega_{0}^{-p e(t) s} \omega^{i(p+1)^{t} s} \\
& =[C: X] \sum_{i=0}^{p a-1-1} \omega_{0}^{s} \omega^{i(p+1)^{t} s} .
\end{aligned}
$$

By (2.10) as $t$ goes from 0 to $p^{\alpha-1}-1$, if $(p+1)^{t}=a p+1, a$ varies from 0 to $p^{\alpha-1}-1$ (modulo $\left.p^{\alpha-1}\right)$. Thus

$$
\begin{aligned}
\sigma_{i}^{H}\left(x^{s}\right) & =[C: X] \sum_{i=0}^{p a-1-1} \omega_{0}^{s} \omega^{i(p+1)^{t} s} \\
& =[C: X] \omega_{0}^{s} \sum_{a=0}^{p a-1-1} \omega^{i(a p+1) s} \\
& =[C: X] \omega_{0}^{s} \omega^{i s} \sum_{a=0}^{p a-1-1}\left(\omega^{i p s}\right)^{a} .
\end{aligned}
$$

If $i \neq 0$ (modulo $\left.p^{\alpha-1}\right)$, since $s \not \equiv 0$ (modulo $\left.p\right)$ and $\alpha \geq 2, \omega^{i s p} \neq 1$. Then

$$
\sum_{a=0}^{p a-1-1}\left(\omega^{i s p}\right)^{a}=\left(1-\omega^{i s p^{a}}\right) /\left(1-\omega^{i s p}\right)=0
$$

since $\omega$ is a $p^{\alpha}$ th root of 1 . 


$$
\text { Since } \begin{array}{r}
\lambda^{H}=\sum_{i=0}^{p^{\alpha}-1} \sigma_{i}^{H}=e\left(\phi_{1}+\cdots+\phi_{p}\right), \\
\sigma_{i}^{H}=\sum_{j=1}^{p} n_{i j} \phi_{j}
\end{array}
$$

for some nonnegative integers $n_{i j}$. Consider $\sigma_{i}^{H}$, for $i \not \equiv 0$ (modulo $p^{\alpha-1}$ ). If $y \in H, \sigma_{i}^{H}(y)=0$ for all $y \notin T(H, \lambda)$ since each $\phi_{j}(y)=0$ by Theorem 1.8. If $y \in T(H, \lambda), y \notin Z$, then $y$ is conjugate to $x^{s} \cdot z$ for some $1 \leq s \leq p-1$ and some $z \in Z$. However

$$
\sigma_{i}^{H}\left(x^{s} \cdot z\right)=\sigma_{i}^{H}\left(x^{s}\right) \cdot \lambda(z)=0 .
$$

Since $\sigma_{i}^{H}$ is a class function, $\sigma_{i}^{H}(y)=0$ for all $y \in H, y \notin Z$. If $y \in Z$, $\sigma_{i}^{H}(y)=[H: X] \lambda(y)$. Hence $\sigma_{i}^{H}$ is a multiple of $\lambda^{H}$ or

$$
\sigma_{i}^{H}=\left(e / p^{\alpha}\right)\left(\phi_{1}+\cdots+\phi_{p}\right)
$$

Thus $n_{i j}=e / p^{\alpha}$ for all $1 \leq j \leq p$ and all $i \not \equiv 0$ (modulo $\left.p^{\alpha-1}\right)$. Let $K=e / p^{\alpha}$. Since $\left.\lambda^{H}\right|_{X}=e\left(\left.\phi_{1}\right|_{X}+\cdots+\left.\phi_{p}\right|_{X}\right)=[H: X] \lambda^{X}=[H: X] \sum_{i=0}^{p^{a}-1} \sigma_{i}$, we have

$$
\begin{aligned}
& \left.\phi_{j}\right|_{X}=\sum_{i=0}^{p^{\alpha}-1} n_{i j} \sigma_{i} \\
& =\sum_{u=0}^{p-1} \sum_{v=0}^{p^{\alpha-1}-1}\left(n_{u p^{a-1}+v, j}\right) \sigma_{u p^{a-1}+\nu} \\
& =\sum_{u=0}^{p-1}\left(n_{u p^{a-1}, j}\right) \sigma_{u p^{\alpha-1}}+\sum_{u=0}^{p-1} \sum_{\nu=1}^{p^{a-1}-1}\left(n_{u p^{a-1}+v, j}\right) \sigma_{u p^{a-1}+v} \\
& =\sum_{u=0}^{p-1}\left(n_{u p^{a-1}, j}\right) \sigma_{u p^{a-1}}+K \sum_{u=0}^{p-1} \sum_{v=1}^{p^{a-1}-1} \sigma_{u p^{a-1}+v} \\
& =\sum_{u=0}^{p-1}\left(n_{u p^{a-1}, j}-K\right) \sigma_{u p^{a-1}}+K \sum_{\nu=0}^{p^{a}-1} \sigma_{v} \text {. }
\end{aligned}
$$

Let $k_{u j}=\left(n_{u p-1, j}\right)-K$. Then (2.14) follows.

To show $H$ is $p$-special, suppose $\sum_{j=1}^{p} m_{j} \phi_{j}(y)=0$ for some $y \in H$ and nonnegative integers $m_{j}$. If $y \notin T(H, \lambda), \phi_{j}(y)=0$ for all $j$ by Theorem 1.8. If $y \in Z$, then

$$
0=\sum_{j=1}^{p} m_{j} \phi_{j}(y)=\sum_{j=1}^{p} m_{j} \cdot e \lambda(y) .
$$

Thus $m_{j}=0$ for all $1 \leq j \leq p$. If $y \in T(H), y \notin Z$, then $y$ is conjugate to $x^{s} \cdot z$ for some $1 \leq s \leq p-1$, and $z \in Z$. Then

$$
\sum_{j=1}^{p} m_{j} \phi_{j}\left(x^{s} \cdot z\right)=0 .
$$


Using (2.14), we find

$$
\begin{aligned}
0 & =\sum_{j=1}^{p} m_{j} \phi_{j}\left(x^{s} \cdot z\right) \\
& =\sum_{j=1}^{p} m_{j}\left(\sum_{u=0}^{p-1} k_{u j} \sigma_{p a-1}\left(x^{s} \cdot z\right)\right)+K\left(\sum_{j=1}^{p} m_{j}\right) \sum_{v=0}^{p^{a}-1} \sigma_{v}\left(x^{s} \cdot z\right) .
\end{aligned}
$$

Since $1 \leq s \leq p-1$,

$$
\sum_{v=0}^{p^{a}-1} \sigma_{v}\left(x^{s} \cdot z\right)=\lambda^{X}\left(x^{s} \cdot z\right)=0 .
$$

Hence

$$
\begin{aligned}
0 & =\sum_{u=0}^{p-1}\left(\sum_{j=1}^{p} m_{j} k_{u j}\right) \sigma_{p a-1}\left(x^{s} \cdot z\right) \\
& =\sum_{u=0}^{p-1}\left(\sum_{j=1}^{p} m_{j} k_{u j}\right) \omega_{0}^{s} \lambda(z) \cdot \omega^{\left.p^{a-1}\right|_{u-s}}
\end{aligned}
$$

and

$$
0=\sum_{u=0}^{p-1}\left(\sum_{j=1}^{p} m_{j} k_{u j}\right) \omega^{p^{a-1} u \cdot s} .
$$

Since each $\omega^{p^{a-1} 1_{u-s}}$ is a $p$ th root of 1 , the above equation implies that $\sum_{j=1}^{p} m_{j} k_{u j}$ $=\sum_{j=1}^{p} m_{j} k_{0 j}$ for all $0 \leq u \leq p-1$. Then

$$
\begin{array}{r}
\left.\sum_{j=1}^{p} m_{j} \phi_{j}\right|_{x}=\sum_{u=0}^{p-1}\left(\sum_{j=1}^{p} m_{j} k_{u j}\right) \sigma_{p a-1}+K\left(\sum_{j=1}^{p} m_{j}\right)\left(\sum_{\nu=0}^{p^{a}-1} \sigma_{v}\right) \\
=\left(\sum_{j=1}^{p} m_{j} k_{0 j}\right)\left(\sum_{u=0}^{p-1} \sigma_{p a-1}\right)+K\left(\sum_{j=1}^{p} m_{j}\right)\left(\sum_{\nu=0}^{p^{\alpha}-1} \sigma_{v}\right) .
\end{array}
$$

For all $1 \leq s \leq p-1$ and $z \in Z, \sum_{j=1}^{p} m_{j} \phi_{j}\left(x^{s} \cdot z\right)=0$. Since $T(H, \lambda)$ $=\cup_{i=0}^{p-1} \mathrm{Cl}\left(x^{i}\right) \cdot Z, \sum_{j=1}^{p} m_{j} \phi_{j}(y)=0$ for all $y \notin Z$. Thus $\sum_{j=1}^{p} m_{j} \phi_{j}$ is a multiple of $\lambda^{H}$ or

$$
\sum_{j=1}^{p} m_{j} \phi_{j}=\left(\sum_{j=1}^{p} m_{j} / p\right)\left(\phi_{1}+\cdots+\phi_{p}\right)
$$

Thus $m_{j}=\left(\sum_{j=1}^{p} m_{j}\right) / p$ or $m_{j}=m_{i}$ for all $i, j$. Hence $H$ is $p$-special.

We can describe the Sylow subgroups of $p$-special groups.

Theorem 2.17. Let $H$ be a p-special group. Then

(a) for any prime $q \neq p$, and any $q$ Sylow subgroup $S_{q}$ of $H, S_{q}$ is of central type with $Z\left(S_{q}\right)=Z \cap S_{q}$ 
(b) If $S_{p}$ is any $p$ Sylow subgroup of $H$, then either $S_{p}$ is of central type with $\left[Z\left(S_{p}\right): Z \cap S_{p}\right]=p$ or $S_{p}$ is p-special with $Z\left(S_{p}\right)=Z \cap S_{p}$.

Proof. Let $\lambda$ be a linear character on $Z$, such that $H$ is p-special with respect to $\lambda$. Let $\lambda^{H}=e\left(\phi_{1}+\cdots+\phi_{p}\right)$ where $\phi_{1}, \ldots, \phi_{p}$ are inequivalent irreducible characters on $H$. Let $q$ be any prime, let $S_{q}$ be a $q$ Sylow subgroup of $H$, and let $R_{q}=S_{q} \cdot Z$. Let

$$
\lambda^{R_{4}}=\gamma_{1}(1) \gamma_{1}+\cdots+\gamma_{s}(1) \gamma_{s}
$$

where $\gamma_{1}, \ldots, \gamma_{s}$ are inequivalent irreducible characters on $R_{q}$ Since $\left.\gamma_{i}\right|_{s_{q}}$ is irreducible, $\gamma_{i}(1)$ is a power of $q$. Since $\gamma_{i}$ is a constituent of $\lambda^{R_{4}}$,

$$
\gamma_{i}^{H}=\sum_{j=1}^{p} k_{i j} \phi_{j}
$$

for some integers $k_{i j}$. Then

$$
\gamma_{i}^{H}(1)=[H: R] \gamma_{i}(1)=\sum_{j=1}^{P} k_{i j} \phi_{j}(1)=e\left(\sum_{j=1}^{P} k_{i j}\right) .
$$

By taking $q$ parts we get

$$
\gamma_{i}(1)=e_{q}\left(\sum_{j=1}^{p} k_{i j}\right)_{q} .
$$

If $q \neq p$, since $e^{2}=[H: Z] / p$, we get

$$
e_{q}^{2}=([H: Z] / p)_{q}=[H: Z]_{q}=\left[R_{q}: Z\right] .
$$

Hence $\gamma_{i}(1)^{2} \geq\left[R_{q}: Z\right]$. However

$$
\gamma_{i}(1)^{2} \leq\left[R_{q}: Z\left(R_{q}\right)\right] \leq\left[R_{q}: Z\right] .
$$

Hence $\gamma_{i}(1)^{2}=\left[R_{q}: Z\left(R_{q}\right)\right], Z\left(R_{q}\right)=Z$, and $R_{q}$ is of central type. Thus $S_{q}$ is of central type and $Z\left(S_{q}\right)=Z \cap S_{q}$.

If $q=p$, then

$$
e_{p}^{2}=([H: Z] / p)_{p}=\left([H: Z]_{p}\right) / p=\left[R_{p}: Z\right] / p .
$$

Since $\gamma_{i}(1)^{2} \leq\left[R_{p}: Z\left(R_{p}\right)\right] \leq\left[R_{p}: Z\right]$ and

$$
\gamma_{i}(1)^{2}=e_{p}^{2}\left(\sum_{i=1}^{p} k_{i j}\right)_{p}^{2}=\left(\left[R_{p}: Z\right] / p\right)\left(\sum_{j=1}^{p} k_{i j}\right)_{p}^{2},
$$

we have that $\left(\sum_{j=1}^{p} k_{i j}\right)_{p}^{2}=1$. Hence

$$
\gamma_{i}(1)^{2}=\left[R_{p}: Z\right] / p \text { for all } i .
$$


Let $e_{p}^{2}=\left[R_{p}: Z\right] / p$. Then $\lambda^{R_{p}}=e_{p}\left(\gamma_{1}+\cdots+\gamma_{p}\right)$. If $Z\left(R_{p}\right) \neq Z,\left[Z\left(R_{p}\right): Z\right]$ must be a power of $p$. Since $\gamma_{i}(1)^{2}=\left[R_{p}: Z\right] / p,\left[Z\left(R_{p}\right): Z\right]=p$. Then $\gamma_{i}(1)^{2}=$ $\left[R_{p}: Z\left(R_{p}\right)\right]$ for each $i$ and $R_{p}$ is of central type. Hence in the case that $Z\left(R_{p}\right) \neq Z, S_{p}$ is of central type with $\left[Z\left(S_{p}\right): Z \cap S_{p}\right]=p$.

Assume $Z\left(R_{p}\right)=Z$. As in the proof of Theorem 2.8, there is an $x \in S_{p}$, such that $T\left(R_{p}, \lambda\right)=\bigcup_{i=0}^{p-1} \mathrm{Cl}_{R_{p}}\left(x^{i}\right) \cdot Z$. Since $\lambda^{R_{p}}=e_{p}\left(\gamma_{1}+\cdots+\gamma_{p}\right)$, if $\lambda$ is faithful on $\left[R_{p}, R_{p}\right] \cap Z$, by Lemma $2.13, R_{p}$ is $p$-special. If $\lambda$ is not faithful on $\left[R_{p}, R_{p}\right] \cap Z$, let $N=\left[R_{p}, R_{p}\right] \cap \operatorname{kernel}(\lambda)$. Let $\bar{R}_{p}=R_{p} / N, \bar{Z}=Z / N, \bar{\lambda}(z N)$ $=\lambda(z)$ for any $z \in z N$, and $\bar{\gamma}_{i}(r N)=\gamma_{i}(r)$ for any $r \in r N$. Then

$$
\bar{\lambda}^{\bar{h}_{p}}=e_{p}\left(\bar{\gamma}_{1}+\cdots+\bar{\gamma}_{p}\right) \text { and } T\left(\bar{R}_{p}, \bar{\lambda}\right)=\bigcup_{i=0}^{p-1} \mathrm{Cl}_{\bar{R}_{p}}\left(\bar{x}^{i}\right) \cdot \bar{Z} \text {. }
$$

By Lemma 2.13, $\bar{R}_{p}$ is $p$-special. Suppose $\sum_{j=1}^{p} m_{j} \gamma_{j}(y)=0$ for some $y \in R_{p}$ and nonnegative integers $m_{j}$. Then

$$
0=\sum_{j=1}^{p} m_{j} \bar{\gamma}_{j}(\bar{y})
$$

and since $\bar{R}_{p}$ is $p$-special, either $\bar{\gamma}_{j}(\bar{y})=0$ for all $j$, or $m_{i}=m_{j}$ for all $i, j$. Hence, either $\gamma_{j}(y)=0$ for all $j$, or $m_{i}=m_{j}$ for all $i, j$ and $R_{p}$ is $p$-special. Thus, if $Z\left(R_{p}\right)=Z$, then $R_{p}$ is $p$-special. Hence, $S_{p}$ is $p$-special with $Z\left(S_{p}\right)=Z \cap S_{p}$.

We can describe simply which of the two possibilities in (b) occurs in the case that $p \neq 2$. Example 2.3 shows that this characterization does not hold when $p=2$.

Corollary 2.18. Let $H$ be a group with center $Z$. Assume $[H, H] \cap Z$ is cyclic and $\lambda$ is a linear character on $Z$, faithful on $[H, H] \cap Z$. Let $T(H)=T(H, \lambda)$ $=\left\{h \in H: h^{-1} \mathrm{Cl}_{H}(h) \cap Z=\{1\}\right\}$. Assume $H$ is p-special with respect to $\lambda$ for some prime $p \neq 2$. If $x \in T(H), x \notin Z$, and $X=\langle x\rangle \cdot Z$, then $X$ is not normal in $H$. If $S$ is a pylow subgroup of $H, R=S \cdot Z$, Let

$$
T(R)=T(R, \lambda)=\left\{r \in R: r^{-1} \mathrm{Cl}_{R}(r) \cap Z=\{1\}\right\} .
$$

Let $x \in T(R), x \notin Z$. Then $R$ is of central type if and only if $X=\langle x\rangle \cdot Z$ is normal in $R$.

Proof. Since $H$ is $p$-special with respect to $\lambda$, by Theorem 2.8 there is $x_{0} \in S$ so that

(a) $T(R)=\bigcup_{i=0}^{-1} \mathrm{Cl}_{R}\left(x_{0}^{i}\right) \cdot Z$;

(b) $T(H)=\cup_{i=0}^{p-1} \mathrm{Cl}_{H}\left(x_{0}^{i}\right) \cdot Z$.

By Lemma 2.10, for all integers $a$ and all $1 \leq i \leq p-1$,

$$
\mathrm{Cl}_{R}\left(x_{0}^{(a p+1) i}\right) \cdot \mathrm{Z}=\mathrm{Cl}_{R}\left(x_{0}^{i}\right) \cdot \mathrm{Z}
$$


and

$$
\mathrm{Cl}_{H}\left(x_{0}^{(a p+1) i}\right) \cdot Z=\mathrm{Cl}_{H}\left(x_{0}^{i}\right) \cdot Z \text {. }
$$

Also $\lambda^{H}=e\left(\phi_{1}+\cdots+\phi_{p}\right)$ where $\phi_{1}, \ldots, \phi_{p}$ are inequivalent irreducible characters on $H$ and $\lambda^{R}=e_{p}\left(\gamma_{1}+\cdots+\gamma_{p}\right)$ where $\gamma_{1}, \ldots, \gamma_{p}$ are inequivalent irreducible characters on $R$.

Let $\alpha$ be the minimum number such that $x_{0}^{p^{\alpha}} \in Z$. If $x \in T(R), x \notin Z$, then $x=r^{-1} x_{0}^{i} r z$ for some $r \in R, z \in Z, 1 \leq i \leq p^{\alpha}-1$, with $i$ relatively prime to $p$. Let $X_{0}=\left\langle x_{0}\right\rangle \cdot Z$. If $X$ is normal in $R$, then $X=X_{0}$, and for each $i$, $\mathrm{Cl}_{R}\left(x_{0}^{i}\right) \subseteq X_{0}$. Hence

$$
T(R) \subseteq X_{0}=X .
$$

Similarly, if there is an $x \in T(H), x \notin Z$, such that $X=\langle x\rangle \cdot Z$ is normal in $H$, then

$$
T(H) \subseteq X
$$

Since $\lambda^{R}$ has $p$ inequivalent irreducible constituents, $T(R) / Z$ contains $p$ distinct conjugacy classes. Hence $\mathrm{Cl}_{R}(x) \cdot Z=\mathrm{Cl}_{R}\left(x^{i}\right) \cdot Z$ only if $i \equiv 1$ (modulo $p$ ). Similarly $\mathrm{Cl}_{H}(x) \cdot Z=\mathrm{Cl}_{H}\left(x^{i}\right) \cdot Z$ only if $i \equiv 1$ (modulo $p$ ). To avoid doing the same argument twice, we prove the following:

(2.19) Let $G$ be a group with center $Z$. Assume $[G, G] \cap Z$ is cyclic and $\lambda$ is a linear character on $Z$, faithful on $[G, G] \cap Z$. Suppose $\lambda^{G}=e\left(\zeta_{1}+\cdots+\zeta_{p}\right)$ where $\zeta_{1}, \ldots, \zeta_{p}$ are inequivalent irreducible characters on $G$. Let

$$
T(G)=\left\{g \in G: g^{-1} \mathrm{Cl}_{G}(g) \cap Z=\{1\}\right\}
$$

and assume $T(G) \neq Z$. If $x \in T(G)$, such that $\mathrm{Cl}_{G}(x) \cdot Z=\mathrm{Cl}_{G}\left(x^{j}\right) \cdot Z$ if and only if $j \equiv 1$ (modulo $p$ ), then $T(G) \nsubseteq\langle x\rangle \cdot Z$.

Proof. Suppose there is an $x \in T(G)$ so that $T(G) \subseteq\langle x\rangle \cdot Z=X$, and $\mathrm{Cl}_{G}(x) \cdot Z=\mathrm{Cl}_{G}\left(x^{j}\right) \cdot Z$ if and only if $j \equiv 1$ (modulo $p$ ).

By Theorem 1.8, $T(G) / Z$ contains $p$ conjugacy classes and thus

$$
T(G)=\bigcup_{i=0}^{p-1} \mathrm{Cl}_{G}\left(x^{i}\right) \cdot Z \text {. }
$$

Let $\alpha$ be the minimum number so that $x^{p^{\alpha}} \in Z$. Let $\omega_{0}$ be a $p^{\alpha}$ th root of $\lambda\left(x^{p^{\alpha}}\right)$ and let $\omega$ be a primitive $p^{\alpha}$ th root of 1 . Define $\sigma_{i}\left(x^{s} \cdot z\right)=\omega_{0}^{s} \omega^{s i} \lambda(z)$. As in the proof of Lemma 2.12, $\lambda^{x}=\sum_{i=0}^{p^{*}-1} \sigma_{i}$. Since $p \neq 2$, by (2.14) there are integers $K$ and $k_{u j}, 0 \leq u \leq p-1,1 \leq j \leq p$, such that

$$
\left.\zeta_{j}\right|_{X}=\sum_{u=0}^{p-1} k_{u j} \sigma_{p-1}+K \sum_{\nu=0}^{p e-1} \sigma_{v}
$$


If $\alpha \neq 1, K=e / p^{\alpha}$ and

$$
\zeta_{j}(1)=e=\left(\sum_{u=0}^{p-1} k_{u j}\right)+K p^{\alpha}=\left(\sum_{u=0}^{p-1} k_{u j}\right)+e .
$$

Hence $\sum_{u=0}^{p-1} k_{u j}=0$. Since $T(G)=\cup_{i=0}^{p-1} \mathrm{Cl}_{G}\left(x^{i}\right) \cdot Z \subseteq X, g^{-1} x^{i} g \in X$ for every $g \in G$ and every $i$. Therefore $X$ is normal in $G$. By Clifford's theorem [4, Theorem 49.2, p. 343] since

$$
\left.\zeta_{j}\right|_{X}=\sum_{u=0}^{p-1} k_{u j} \sigma_{p a-1}+K \sum_{\nu=0}^{p^{a}-1} \sigma_{v}
$$

for every $0 \leq u \leq p-1$, either $k_{u j}+K=0$ or $k_{u j}=0$. Since $\sum_{u=0}^{p-1} k_{u j}=0$, $k_{u j}=0$ for every $u$, and every $j$. Then

$$
\left.\zeta_{j}\right|_{x}=K \sum_{v=0}^{p^{e}-1} \sigma_{v}
$$

and $\zeta_{j}(x)=0$ for every $j$. By Theorem $1.8, x \notin T(G)$ which is a contradiction. Therefore $\alpha=1$. If $g \in G, g^{-1} x g \in X$ and hence $g^{-1} x g=x^{i} \cdot z$. Since $\mathrm{Cl}_{G}(x)$ $\cdot Z=\mathrm{Cl}_{G}\left(x^{j}\right) \cdot Z$ only if $j \equiv 1$ (modulo $p$ ), $i \equiv 1$ (modulo $p$ ). Since $\alpha=1$, we can assume $i=1$. Since $x \in T(G)$, and $g^{-1} x g=x \cdot z, z=1$. Therefore for all $g \in G, g^{-1} x g=x$ and $x \in Z(G)$. Since $Z \subseteq T(G) \subseteq X \subseteq Z(G)=Z, T(G)$ $=Z$, which contradicts the hypothesis. This completes the proof of (2.19).

Returning to the proof of Corollary 2.18, we have that if there is an $x \in T(H)$, $x \notin Z$ such that $\langle x\rangle \cdot Z$ is normal in $H$, then $T(H) \subseteq\langle x\rangle \cdot Z$ and by (2.19), $T(H)=Z(H)$ which is impossible, since $x \notin Z=Z(H)$. If there is an $x \in T(R), x \notin Z$, such that $\langle x\rangle \cdot Z$ is normal in $H$, then $T(R) \subseteq\langle x\rangle \cdot Z$ and by (2.19), $T(R)=Z(R)$. If $T(R)=Z(R)$, then $Z(R) \neq Z$ and $R$ is of central type with $[Z(R): Z]=p$.

If $R$ is of central type, then $[Z(R): Z]=p$. Since $\lambda^{R}$ has $p$ inequivalent irreducible constituents, by Theorem $1.8, T(R) / Z$ contains $p$ conjugacy classes of $R / Z$. Clearly, $Z(R) \subseteq T(R)$. Thus $Z(R)=T(R)$. If $x \in T(R), x \notin Z$, then $X=\langle x\rangle \cdot Z=Z(R)$ and $X$ is normal in $R$.

There is a close relationship between the structure of $T(H, \lambda)$ and the structure of the Sylow subgroups of $H$.

Theorem 2.20. Let $H$ be a group with center $Z$ and assume that $[H, H] \cap Z$ is cyclic. Let

$$
T(H)=\left\{x \in H: x^{-1} \mathrm{Cl}_{H}(x) \cap Z=\{1\}\right\} .
$$

Let $q$ be any prime and let $S$ be any $q$ Sylow subgroup of $H$. Then $S$ is of central type with $Z(S)=S \cap Z$ if and only if $T(H) / Z$ contains no element of order a power of $q$. 
Proof. Let $S$ be a $q$ Sylow subgroup of $H$, and suppose $S$ is of central type with $Z(S)=S \cap Z$. If $R=S \cdot Z$, then $R$ is of central type and $Z(R)=Z$. Let $x$ be any element of $H, x \notin Z$ so that $x Z$ has order a power of $q$. Since all $q$ Sylow subgroups of $H$ are conjugates, there is a conjugate $R^{\prime}$ of $R$ so that $x \in R^{\prime}$. Since $[H, H] \cap Z$ is cyclic, $\left[R^{\prime}, R^{\prime}\right] \cap Z$ is cyclic. Since $R^{\prime}$ is isomorphic to $R, R^{\prime}$ is of central type. By Theorem 4 of [6], $T\left(R^{\prime}\right)=Z\left(R^{\prime}\right)$. Since $Z\left(R^{\prime}\right)=Z$ and $x \notin Z$, there is an $r \in R^{\prime}$ so that $r^{-1} x r=x \cdot z, z \neq 1, z \in Z$. Thus $x \notin T(H)$.

Conversely, suppose $T(H) / Z$ contains no element of order a power of $q$. Let $\lambda$ be a linear character on $Z$, such that $\lambda$ is faithful on $[H, H] \cap Z$. Let $\lambda^{H}=\phi_{1}(1) \phi_{1}+\cdots+\phi_{t}(1) \phi_{t}$ where $\phi_{1}, \ldots, \phi_{t}$ are inequivalent irreducible characters on $H$. Let $S$ be any $q$ Sylow subgroup of $H, R=S \cdot Z$ and let $\lambda^{R}=\gamma_{1}(1) \gamma_{1}+\cdots+\gamma_{s}(1) \gamma_{s}$ where $\gamma_{1}, \ldots, \gamma_{s}$ are inequivalent irreducible characters on $R$. Since $\gamma_{j}$ is a constituent of $\lambda^{R}$ and $\left.\lambda^{H}\right|_{R}=[H: R] \lambda^{R}$,

$$
\gamma_{j}^{H}=\sum_{i=1}^{\ell} k_{i j} \phi_{i}, \quad 1 \leq j \leq s,
$$

and

$$
\left.\phi_{i}\right|_{R}=\sum_{j=1}^{s} k_{i j} \gamma_{j}, \quad 1 \leq i \leq t,
$$

for some nonnegative integers $k_{i j}$. Let $K_{i}$ be the greatest common divisor of $k_{i j}$, $1 \leq j \leq s$, and let $k_{i j}=K_{i} k_{i j}^{\prime}$. Let $M$ be the least common multiple of $\phi_{i}(1)$, $1 \leq i \leq t$.

Since $T(H) / Z$ does not contain a $q$ element, if $r \in R, r \notin Z$, then $r \notin T(H)$ and hence, by Theorem 1.8, $\phi_{i}(r)=0$ for all $i$. Thus for all $r \in R$, and all $i, u$

$$
\left(M / \phi_{i}(1)\right) \phi_{i}(r)=\left(M / \phi_{u}(1)\right) \phi_{u}(r)
$$

or

$$
\left.\left(M / \phi_{i}(1)\right) \phi_{i}\right|_{R}=\left.\left(M / \phi_{u}(1)\right) \phi_{u}\right|_{R}
$$

Thus, by equation (2.21), $\left(M / \phi_{i}(1)\right) k_{i j}=\left(M / \phi_{u}(1)\right) k_{u j}$ for all $i, j, u$, or $\left(M / \phi_{i}(1)\right) K_{i} k_{i j}^{\prime}=\left(M / \phi_{u}(1)\right) K_{u} k_{u j}^{\prime}$. Thus

$$
k_{i j}^{\prime}=\left(\phi_{i}(1) K_{u} / \phi_{u}(1) K_{i}\right) k_{u j}^{\prime} .
$$

Since $k_{i j}^{\prime}, 1 \leq j \leq s$, have no common divisors, and $k_{u j}^{\prime}, 1 \leq j \leq s$, have no common divisors

$$
\phi_{i}(1) K_{u}=\phi_{u}(1) K_{i} \text { or } \phi_{u}(1) / K_{u}=\phi_{i}(1) / K_{i} \text { for all } i, u \text {. }
$$

From equation (2.22), we have

$$
\left.\phi_{i}\right|_{R}=K_{i} \sum_{n=1}^{s} k_{i j}^{\prime} \gamma_{j}
$$


and hence $K_{i}$ divides $\phi_{i}(1)$. Thus

$$
\phi_{i}(1) / K_{i}=L
$$

for all $i$ where $L$ is an integer independent of $i$. For every $i$,

$$
\sum_{j=1}^{s} k_{i j}^{\prime} \gamma_{j}(r)=0 \text { if } r \notin Z
$$

and

$$
\sum_{j=1}^{s} k_{i j}^{\prime} \gamma_{j}(r)=L \lambda(r) \text { if } r \in Z
$$

Hence $\sum_{j=1}^{s} k_{i j}^{\prime} \gamma_{j}$ is a multiple of $\lambda^{R}$ or

$$
\sum_{j=1}^{s} k_{i j}^{\prime} \gamma_{j}=(L /[R: Z])\left(\gamma_{1}=(1) \gamma_{1}+\cdots+\gamma_{s}(1) \gamma_{s}\right) .
$$

Thus $k_{i j}^{\prime}=(L /[R: Z]) \gamma_{j}(1)$ for all $i, j$. Let $q^{\alpha}$ be the minimum value of $\gamma_{j}(1)$, $1 \leq j \leq s$. Then $q^{\alpha} L /[R: Z]$ is an integer. If $[R: Z]=q^{\beta}$, then $L$ is divisible by $q^{\beta-\alpha}$. By equation (2.23), $\phi_{i}(1)$ is divisible by $q^{\beta-\alpha}$ for all $i$. By equation (2.21),

$$
\gamma_{j}^{H}(1)=[H: R] \gamma_{j}(1)=\sum_{i=1}^{\ell} k_{i j} \phi_{i}(1), \quad 1 \leq j \leq s .
$$

Since $[H: R]$ is relatively prime to $q$, and each $\phi_{i}(1)$ is divisible by $q^{\beta-\alpha}$, each $\gamma_{j}(1)$ is divisible by $q^{\beta-\alpha}$. For some $j, q^{\alpha}=\gamma_{j}(1)$, and hence $\alpha \geq \beta-\alpha$ or $2 \alpha \geq \beta$. However

$$
\gamma_{j}(1)^{2} \leq[R: Z(R)] \leq[R: Z]=q^{\beta} .
$$

Therefore $2 \alpha \leq \beta, \beta=2 \alpha$,

$$
\gamma_{j}(1)^{2}=[R: Z(R)] \text { and } Z(R)=Z .
$$

Hence $R$ is of central type and $Z(R)=Z$. Thus $S$ is also of central type with $Z(S)=S \cap Z$.

We can now characterize p-special groups in terms of the structure of the group. Notice that this theorem is the converse of Theorem 2.8, in the case that $\lambda$ is faithful on $[H, H] \cap Z$.

Theorem 2.24. Let $H$ be a group with center $Z$ and assume that $[H, H] \cap Z$ is cyclic. Let

$$
T(H)=\left\{x \in H: x^{-1} \mathrm{Cl}_{H}(x) \cap Z=\{1\}\right\} .
$$

Let $S$ be any $p$ Sylow subgroup of $H, R=S \cdot Z$, and let

$$
T(R)=\left\{x \in R: x^{-1} \mathrm{Cl}_{R}(x) \cap Z=\{1\}\right\} .
$$


Assume there is an $x \in S$ such that

(a) $T(R)=\bigcup_{i=0}^{p-1} \mathrm{Cl}_{R}\left(x^{i}\right) \cdot Z$.

(b) $T(H)=\cup_{i=0}^{p-1} \mathrm{Cl}_{H}\left(x^{i}\right) \cdot Z$.

(c) For $i \neq 0$ (modulo $p$ ), $\mathrm{Cl}_{H}\left(x^{i}\right) \cdot Z=\mathrm{Cl}_{H}\left(x^{j}\right) \cdot Z$ if and only if $i \equiv j$ (modulo p).

Then $H$ is p-special.

Proof. Let $\lambda$ be a linear character on $Z$, with $\lambda$ faithful on $[H, H] \cap Z$, Let $\lambda^{H}=\phi_{1}(1) \phi_{1}+\cdots+\phi_{t}(1) \phi_{t}$ where $\phi_{1}, \ldots, \phi_{t}$ are inequivalent irreducible characters on $H$.

Let $q$ be a prime, $q \neq p$, let $S_{q}$ be a $q$ Sylow subgroup of $H$, and let $R_{q}=S_{q} \cdot Z$. By Theorem 2.20, $S_{q}$ and $R_{q}$ are of central type since (b) implies that $T(H) / Z$ contains no $q$ elements. Also $T\left(R_{q}\right)=Z$. Since $T\left(R_{q}\right) / Z$ contains only one conjugacy class, by Theorem $1.8, \lambda^{R_{q}}$ has only one irreducible constituent. Let $\lambda^{R_{q}}=\zeta_{q}(1) \zeta_{q}$. Then

$$
\lambda^{H}=\zeta_{q}(1) \zeta_{q}^{H}=\phi_{1}(1) \phi_{1}+\cdots+\phi_{t}(1) \phi_{t} .
$$

Thus $\zeta_{q}(1)$ divides $\phi_{i}(1)$ for each $i$. Since $\zeta_{q}(1)^{2}=\left[R_{q}: Z\right]$, each $\phi_{i}(1)^{2}$ is divisible by $\left[R_{q}: Z\right]=[H: Z]_{q}$, where $[H: Z]_{q}$ denotes the $q$ factor of $[H: Z]$.

Let $S$ be any $p$ Sylow subgroup of $H$ and let $R=S \cdot Z$. Since $T(R) / Z$ contains $p$ conjugacy classes, $\lambda^{R}$ has $p$ inequivalent irreducible constituents by Theorem 1.8. Since each irreducible constituent of $\lambda^{R}$ has degree a power of $p$, and their squares add up to $[R: Z]$ which is also a power of $p$, all irreducible constituents of $\lambda^{R}$ must have the same degree. Let $e_{p}$ be this common degree. Then $\lambda^{R}=e_{p}\left(\gamma_{1}+\cdots+\gamma_{p}\right)$ where $\gamma_{1}, \ldots, \gamma_{p}$ are inequivalent irreducible characters on $R$. Then

$$
\lambda^{H}=e_{p}\left(\gamma_{1}^{H}+\cdots+\gamma_{p}^{H}\right)=\phi_{1}(1) \phi_{1}+\cdots+\phi_{t}(1) \phi_{t} .
$$

Thus $e_{p}$ divides each $\phi_{i}(1)$. Since $e_{p}^{2}=[R: Z] / p$, each $\phi_{i}^{2}(1)$ is divisible by $[R: Z] / p=[H: Z]_{p} / p$, where $[H: Z]_{p}$ denotes the $p$ part of $[H: Z]$. Then each $\phi_{i}(1)^{2}$ is divisible by $[H: Z]_{q}$ for all primes $q \neq p$ and by $[H: Z]_{p} / p$ or $\phi_{i}(1)^{2}$ is divisible by $[H: Z] / p$. Since

$$
[H: Z]=\sum_{i=1}^{t} \phi_{i}(1)^{2}
$$

$t \leq p$. Since $t(H)=\bigcup_{i=0}^{p-1} \mathrm{Cl}_{H}\left(x^{i}\right) \cdot Z$ and $\mathrm{Cl}_{H}\left(x^{i}\right) \cdot Z \neq \mathrm{Cl}_{H}\left(x^{j}\right) \cdot Z$ for $1 \leq i$, $j \leq p-1$ by (c), $T(H) / Z$ contains $p$ conjugacy classes. Hence, by Theorem 1.8 , $\lambda^{H}$ has $p$ inequivalent irreducible constituents. Hence $t=p$, and if $e^{2}=[H$ : $Z] / p$, then $\phi_{i}(1)=e$ for all $i$ and

$$
\lambda^{H}=e\left(\phi_{1}+\cdots+\phi_{p}\right) .
$$

By Lemma $2.13 H$ is $p$-special. 
The condition on $[H, H] \cap Z$ can be dropped and we have the following theorem, which is the converse of Theorem 2.8 in all cases.

Theorem 2.25. Let $H$ be a group with center $Z$. Let $\lambda$ be a linear character on $Z$ and let

$$
T(H, \lambda)=\left\{x \in H: x^{-1} \mathrm{Cl}_{H}(x) \cap Z \subseteq \operatorname{kernel}(\lambda)\right\} .
$$

Let $S$ be any $p$ Sylow subgroup of $H$ and let $R=S \cdot Z$. Let

$$
T(R, \lambda)=\left\{x \in R: x^{-1} \mathrm{Cl}_{R}(x) \cap Z \subseteq \operatorname{kernel}(\lambda)\right\} .
$$

Assume there is $x \in S$ such that

(a) $T(R, \lambda)=\bigcup_{i=0}^{p-1} \mathrm{Cl}_{R}\left(x^{i}\right) \cdot Z$.

(b) $T(H, \lambda)=\bigcup_{i=0}^{p-1} \mathrm{Cl}_{H}\left(x^{i}\right) \cdot Z$. p).

(c) For $i \not \equiv 0$ (modulo $p$ ), $\mathrm{Cl}_{H}\left(x^{i}\right) \cdot Z=\mathrm{Cl}_{H}\left(x^{j}\right) \cdot Z$ if and only if $i \equiv j$ (modulo

Then $H$ is p-special with respect to $\lambda$.

Proof. Let $N=[H, H] \cap \operatorname{kernel}(\lambda)$. Let $\bar{H}=H / N, \bar{R}=R / N, \bar{x}=x N$, and $\bar{\lambda}(z N)=\lambda(z)$ for any $z \in z N$. Then

(a') $\overline{T(R, \lambda)}=T(\bar{R}, \bar{\lambda})=\bigcup_{i=0}^{p-1} \mathrm{Cl}_{\bar{R}}\left(\bar{x}^{i}\right) \cdot \bar{Z}$.

(b') $\overline{T(H, \lambda)}=T(\bar{H}, \bar{\lambda})=\bigcup_{i=0}^{p-1} \mathrm{Cl}_{\bar{H}}\left(\bar{x}^{i}\right) \cdot \bar{Z}$.

(c') For $i \not \equiv 0$ (modulo $p$ ), $\mathrm{Cl}_{\bar{H}}\left(\bar{x}^{i}\right) \cdot \bar{Z}=\mathrm{Cl}_{\bar{H}}\left(\bar{x}^{j}\right) \cdot \bar{Z}$ if and only if $i \equiv j$ (modulo $p$ ).

By Theorem 2.24, $\bar{H}$ is $p$-special and

$$
\bar{\lambda}^{H}=e\left(\zeta_{1}+\cdots+\zeta_{p}\right)
$$

where $\zeta_{1}, \ldots, \zeta_{p}$ are inequivalent irreducible characters on $\bar{H}$. Let $\lambda^{H}=\phi_{1}(1) \phi_{1}$ $+\cdots+\phi_{t}(1) \phi_{t}$ where $\phi_{1}, \ldots, \phi_{t}$ are inequivalent irreducible characters on $H$. If $x \in N$, then $\phi_{i}(x)=\phi_{i}(1) \lambda(x)=\phi_{i}(1)$. Define $\bar{\phi}_{i}$ by $\bar{\phi}_{i}(x N)=\phi_{i}(x)$ for any $x \in x N$. Then

$$
\bar{\lambda}^{\bar{H}}=\bar{\phi}_{1}(1) \bar{\phi}_{1}+\cdots+\bar{\phi}_{t}(1) \bar{\phi}_{t}=e\left(\zeta_{1}+\cdots+\zeta_{p}\right) .
$$

Hence $\bar{\phi}_{i}(1)=\phi_{i}(1)=e$ for every $i, t=p$, and by relabeling if necessary $\bar{\phi}_{i}=\zeta_{i}, 1 \leq i \leq t$. Suppose $\sum_{i=1}^{p} m_{i} \phi_{i}(y)=0$ for some $y \in H$ and nonnegative integers $m_{i}$. If $\bar{y}=y N$, then $\sum_{i=1}^{p} m_{i} \bar{\phi}_{i}(\bar{y})=0$. Since $\bar{H}$ is $p$-special, either $\bar{\phi}_{i}(\bar{y})=0$ for all $i$, or $m_{i}=m_{j}$ for all $i, j$. Hence either $\phi_{i}(y)=0$ for all $i$ or $m_{i}=m_{j}$ for all $i, j$. Hence $H$ is $p$-special with respect to $\lambda$.

We can rewrite Theorems 2.8 and 2.25 in a slightly different form.

Corollary 2.26. Let $H$ be a group with center $Z$. Let $\lambda$ be a linear character on $Z$. Let

$$
T(H, \lambda)=\left\{x \in H: x^{-1} \mathrm{Cl}_{H}(x) \cap Z \subseteq \operatorname{kernel}(\lambda)\right\}
$$


Let $S$ be any $p$ Sylow subgroup of $H$, let $R=S \cdot Z$ and let

$$
T(R, \lambda)=\left\{x \in R: x^{-1} \mathrm{Cl}_{R}(x) \cap Z \subseteq \operatorname{kernel}(\lambda)\right\} .
$$

Then $H$ is p-special if and only if

(a) every element of $T(H, \lambda) / Z$ has order a power of $p$ and $T(H, \lambda) / Z$ consists of $p$ conjugacy classes of $H / Z$, and

(b) $T(R, \lambda) / Z$ consists of $p$ conjugacy classes of $R / Z$.

Proof. If $H$ is p-special, then conditions (a) and (b) follow at once from Theorem 2.8.

Suppose conditions (a) and (b) hold. Let $x \in T(R, \lambda), x \notin Z$. Then as in the proof of Lemma 2.11, $x^{i} \in T(R, \lambda)$ for all $1 \leq i \leq p-1$. Since $R / Z$ is a $p$ group, $\mathrm{Cl}_{R}\left(x^{i}\right) \cdot Z \neq \mathrm{Cl}_{R}\left(x^{j}\right) \cdot Z$ for $i \neq j, 1 \leq i, j \leq p-1$. Since $\cup_{i=0}^{p-1} \mathrm{Cl}_{R}\left(x^{i}\right)$ - $Z \subseteq T(R, \lambda)$ and $T(R, \lambda) / Z$ contains only $p$ conjugacy classes of $R / Z$, we have

$$
T(R, \lambda)=\bigcup_{i=0}^{p-1} \mathrm{Cl}_{R}\left(x^{i}\right) \cdot Z .
$$

Let $y \in T(H, \lambda), y \notin Z$. Since $y Z$ has order a power of $p$ and all $p$ Sylow subgroups of $H$ are conjugate, $\mathrm{Cl}_{H}(y) \cap R \neq \varnothing$. Let $\boldsymbol{y}^{\prime} \in \mathrm{Cl}_{H}(y) \cap R$. Since $y^{\prime} \in T(H, \lambda) \cap R, y^{\prime} \in T(R, \lambda)$. Since $y^{\prime} \notin Z, y^{\prime} \in \mathrm{Cl}_{R}\left(x^{i}\right) \cdot Z$ for some $i$. Then

$$
\mathrm{Cl}_{R}\left(y^{\prime}\right) \cdot Z=\mathrm{Cl}_{R}\left(x^{i}\right) \cdot Z \text { and } \mathrm{Cl}_{H}\left(y^{\prime}\right) \cdot Z=\mathrm{Cl}_{H}\left(x^{i}\right) \cdot Z \text {. }
$$

Since $y \in \mathrm{Cl}_{H}\left(y^{\prime}\right) \cdot Z, y \in \mathrm{Cl}_{H}\left(x^{i}\right) \cdot Z$. Thus for every $y \in T(H, \lambda), y \notin Z$,

$$
y \in \bigcup_{i=0}^{p-1} \mathrm{Cl}_{H}\left(x^{i}\right) \cdot Z .
$$

Hence $T(H, \lambda) \subseteq \cup_{i=0}^{p-1} \mathrm{Cl}_{H}\left(x^{i}\right) \cdot Z$. Since $T(H, \lambda) / Z$ consists of $p$ conjugacy classes of $H / Z$,

$$
T(H, \lambda)=\bigcup_{i=0}^{p-1} \mathrm{Cl}_{H}\left(x^{i}\right) \cdot Z
$$

and $\mathrm{Cl}_{H}\left(x^{i}\right) \cdot Z \neq \mathrm{Cl}_{H}\left(x^{j}\right) \cdot Z, i \neq j, 1 \leq i, j \leq p-1$. By Lemma 2.11 for all integers $a$ and $i \neq 0$ (modulo $p$ ),

$$
\mathrm{Cl}_{H}\left(x^{(a p+1) i}\right) \cdot Z=\mathrm{Cl}_{H}\left(x^{i}\right) \cdot Z \text {. }
$$

Hence, for $i \not \equiv 0$ (modulo $p)$,

$$
\mathrm{Cl}_{H}\left(x^{i}\right) \cdot Z=\mathrm{Cl}_{H}\left(x^{j}\right) \cdot Z
$$

if and only if $i \equiv$ (modulo $p$ ). Thus $H$ is p-special by Theorem 2.25. 
A word of caution is in order here. One might be tempted to replace (a) of Theorem 2.25 by the statement that either $R$ is of central type with $[Z(R): Z]$ $=p$ or $R$ is $p$-special with $Z=Z(R)$. However, these statements are not equivalent. If $R$ is $p$-special with $Z=Z(R)$, then by Theorem 2.8, $T(R, \lambda)$ $=\bigcup_{i=0}^{p-1} \mathrm{Cl}_{R}\left(x^{i}\right) \cdot Z$ for some $x \in S$, the $p$ Sylow subgroup of $R$. However, if $R$ is of central type with $[Z(R): Z]=p$, it does not follow that $T(R, \lambda)$ $=\bigcup_{i=0}^{p-1} \mathrm{Cl}_{R}\left(x^{i}\right) \cdot Z$ for some $x$, or even that $T(R, \lambda) / Z$ consists of $p$ conjugacy classes of $R / Z$.

Example 2.27. Let $S=\left\langle x, y, z_{0}\right| x^{3}=y^{3}=z_{0}^{3}=1, y^{-1} x y=x z_{0}, y^{-1} z_{0} y$ $\left.=z_{0}, x^{-1} z_{0} x=z_{0}\right\rangle$ and assume $S$ is the $p$ Sylow subgroup of a group $H$ with center $Z$. Let $R=S \cdot Z$, and let $\lambda$ be a linear character on $Z$. Let $\omega$ be a primitive cube root of 1 , and define $\sigma_{i}\left(z_{0}^{s} \cdot z\right)=\lambda(z) \omega^{s i}$, where $z \in Z$. It can be shown that $\sigma_{i}$ is independent of the way elements of $Z(R)=\left\langle z_{0}\right\rangle \cdot Z$ are represented and

$$
\lambda^{Z(R)}=\sigma_{0}+\sigma_{1}+\sigma_{2}
$$

Then

$$
T(R, \lambda)=\left\{x \in R: x^{-1} \mathrm{Cl}_{R}(x) \cap Z \subseteq \operatorname{kernel}(\lambda)\right\}=R .
$$

However, for $i \neq 0$

$$
T\left(R, \sigma_{i}\right)=\left\{x \in R: x^{-1} \mathrm{Cl}_{R}(x) \cap Z(R) \subseteq \operatorname{kernel}\left(\sigma_{i}\right)\right\}=Z(R) .
$$

Hence, for $i=1$ or $i=2, \sigma_{i}^{R}$ has only one irreducible constituent by Theorem 1.8. If $\sigma_{i}^{R}=\zeta_{i}(1) \zeta_{i}$, then $\zeta_{i}(1)^{2}=[R: Z(R)]$ and $R$ is of central type. However, by Theorem 1.8, $\sigma_{0}^{R}$ has 9 inequivalent irreducible constituents. Therefore $\lambda^{R}$ has a total of 11 inequivalent irreducible constituents and $T(R, \lambda) / Z$ consists of 11 conjugacy classes of $R / Z$.

\section{BIBLIOGRAPHY}

1. F. R. DeMeyer, Galois theory in separable algebras over commutative rings, Illinois J. Math. 10 (1966), 287-295.

2.-, Groups with an irreducible character of large degree are solvable, Proc. Amer. Math. Soc. 25 (1970), 615-617.

3. F. R. DeMeyer and G. J. Janusz, Finite groups with an irreducible representation of large degree, Math. Z. 108 (1969), 145-153.

4. C. W. Curtis and I. Reiner, Representation theory of finite groups and associative algebras, Pure and Appl. Math., vol. 11, Interscience, New York, 1962. MR 26 \#2519.

5. B. Huppert, Endliche Gruppen I, Die Grundlehren der math. Wissenschaften, Band 134, SpringerVerlag, Berlin, 1967. MR 37 \#302.

6. Herbert Pahling, Gruppen mit irreduziblen Darstellungen hohen Grades, Mitt. Math. Sem. Giessen 85 (1970), 27-44. MR 41 \#8537.

Department of Mathematics, Colorado State University, Fort Coluns, Colorado 80521

Current address: Box 205, Jacksonville University, Jacksonville, Florida 32211 